\title{
Decadal oscillations in a simplified coupled model due to unstable interactions between zonal winds and ocean gyres
}

\author{
Olivier Arzel*, and Thierry Huck
}

Laboratoire de Physique des Océans (UMR 6523 CNRS IFREMER UBO), Université de Bretagne Occidentale, UFR Sciences F302, 6 avenue Le Gorgeu, B.P. 809, 29285, Brest, France

*oarzel@univ-brest.fr Tel.: +33-298-016509; fax: +33-298-016468.

\begin{abstract}
A simplified coupled ocean-atmosphere model, consisting of a one-layer bidimensional ocean model and a one-layer unidimensional energy balance atmospheric model [J. Clim. 13 (2000) 232] is used to study the unstable interactions between zonal winds and ocean gyres. In a specific range of parameters, decadal variability is found. Anomalies, quite homogeneous zonally, show smallscale wavelength in latitude: perturbations emerge and grow at the southern limb of the intergyre boundary and propagate southward before decaying. The wind stress anomalies are proportional to the meridional gradient of the atmospheric temperature anomalies: this ratio acts as a positive amplification factor, as confirmed by a parameter sensitivity analysis. Assuming zonally-averaged anomalies harmonic in the meridional direction, a very simple analytical model for the perturbations is derived, based on forced Rossby wave adjustment of the western boundary current and its associated anomalous heat transport: it accounts for the scale selection, the growth and the southward propagation of sea surface temperature anomalies in the subtropical gyre. The latter is not only due to the slow advection by the mean current, but to a prevailing mechanism of self-advecting coupled oceanic and atmospheric waves, out of phase in latitude. Relevance to the observational record is discussed.
\end{abstract}

Keywords: Ocean circulation; Air-sea coupling; Air-sea interaction; Ocean-atmosphere system 


\section{Introduction}

Interannual to interdecadal climate variability has been reported in several observations over the past decades (Folland et al., 1984; Levitus, 1989; Ghil and Vautard, 1991; Trenberth, 1990; Tanimoto et al., 1993, among many others). In the North Pacific and South Atlantic, the observed large-scale variability, characterized by covarying anomalies of sea surface temperature (SST) and sea level pressure (SLP), may be due to interactions between surface winds and oceanic gyres. In the ECHO coupled model, Latif and Barnett (1994, 1996) show how the mid-latitude SSTs over the Kuroshio extension strongly interact with the Aleutian low-pressure system to give rise to a 20 yr mode: They suggest that such a period results from the oceanic adjustment, through baroclinic Rossby waves, to wind-stress changes - see also Goodman and Marshall (1999) for a mechanistic approach. In the Hamburg ECHAM/LSG coupled model, Robertson (1996) obtains 18 yr oscillations, and shows similarities in the mode period and structure with those described by Latif and Barnett (1994). In the South Atlantic, the data analysis of Venegas et al. (1998) reveals the existence of coupled SST-SLP interdecadal fluctuations with a period around $20 \mathrm{yr}$, where the horizontal advection of heat in the subtropical gyre and surface heat fluxes are proposed as the dominant physical processes.

Of course several mechanisms have been proposed to explain such decadal variability, as reviewed in Latif (1998) for instance. Various authors have discussed the possible role of stochastic atmospheric forcing in addition to coupling in the excitation of climate variability on decadal time scales in simple ocean-atmosphere models of the North Pacific. Jin (1997) proposed a theory for the interdecadal variability which is produced by both coupling and temporal white noise wind stress and heat flux forcing. Weng and Neelin (1998) argue that interdecadal variability is due to non-local coupled feedback associated with a specific SST pattern in the mid-latitudes, while the stochastic forcing feeds the variance. Sura et al. (1999) show that both the eddy activity along the storms tracks, with its inhomogeneous structure of stochastic wind forcing, and the coupling, are the two key factors exciting an oceanic mode leading to decadal variability. 
To understand such feedback between eddy activity and oceanic currents, Cessi (2000) (C00 hereafter) proposed a simple coupled model, consisting in an unidimensional atmospheric energy balance model with a wind parameterization based on momentum transport by atmospheric perturbations (Green, 1970), coupled to a reduced-gravity ocean model. She obtained regular oscillations of period $18 \mathrm{yr}$, and suggested that the westward propagating oceanic baroclinic Rossby waves across the basin are a key element to the variability period. Moreover she proposed that the sea surface temperature anomalies generated south of the intergyre boundary are advected by the mean surface current around the subtropical gyre. Primeau and Cessi (2001) have reformulated this model within planetary geostrophic equations in spherical coordinates, and show that the mechanism for generating oscillations remains the same. Nevertheless, a major difference is that the SST anomalies are preferentially advected around the subpolar gyre. Following this work, Gallego and Cessi (2000) (GC00 hereafter) built an idealized model which reproduces some features of Cessi's model, and confirmed that the propagation of baroclinic Rossby waves explains the decadal period - however, we found surprising that the scale of the perturbations is much larger than in the original model, while the associated wind stress anomalies have the opposite sign!

The motivation of the present work arose from these intriguing differences between $\mathrm{C} 00$ and GC00 oscillations. First, we analyze the role of planetary waves in the adjustment of the ocean model to wind-stress changes. In a closed domain, the adjustment process results from the superposition of the forced and long baroclinic Rossby waves, the properties of which were examined by LaCasce (2000). He shows that the forced waves can change the apparent phase speed of baroclinic Rossby waves in ocean basins of finite width. We show that, with only time dependent wind forcing, the free baroclinic Rossby wave does not play a significant role close to the western boundary, but the forced wave does. This suggests that the free Rossby waves are not essential to the western boundary current fluctuations, and therefore also not to the temperature variability.

The second step is to understand the growth, scale selection and southward propagation of the SST anomalies in C00, using an approach similar to GC00. Given the zonal 
structure of ocean temperature and streamfunction anomalies, we derive a zonally-averaged analytical model after several simplifications. We show that the southward propagation of SST anomalies is mainly due to a coupled air-sea mode, rather than the mean advection. We finally obtain a single wave-like equation which exhibits a coupled mode of decadal period and a maximum growth rate for wavelength close to the size of the anomalies in the fully coupled model. The resulting basic mechanism we propose is based on the following processes: The meridional wavelength is set by the maximum response of wind stress to temperature anomalies; The western boundary current fluctuations result from the forced Rossby wave response to varying winds; The associated heat transport controls the growth of SST perturbations; Tendency terms for ocean temperature and streamfunction lead to a coherent southward propagation of the whole structure.

The paper is structured as follow: In section 2, the oceanic and atmospheric components of the model are described, and a scaling of the perturbed momentum equation relating the surface wind stress anomaly to surface atmospheric temperature anomaly is derived. In

section 3, the mean state and decadal variability are briefly detailed, the relative influence of interactive wind-stress and heat flux on the variability is assessed, and the adjustment process of the ocean model to changing winds is analyzed. A simple analytical model for zonally-averaged anomalies is derived in section 4, that accounts for the scale, growth and southward propagation of temperature anomalies. In section 5, a parameter sensitivity analysis is performed to validate the simplified model (specifically the amplification parameter responsible for the oscillation) and estimate the robustness of the variability. Discussion and conclusion are given in section 6.

\section{The coupled model}

The model geometry is the same as in C00: It consists in one hemisphere with a single rectangular ocean basin (Fig. 1). Both the atmosphere and the ocean extend from equator $(y=0)$ to pole $\left(y=L_{y}\right)$. The atmosphere is a zonally-averaged single layer of thickness $D$, with a stratification $S$ in potential temperature $\theta$, in energy balance: surface wind stress 
is diagnosed through Green (1970) eddy parameterization. The longitudinal extension of the atmosphere (ocean) is $L_{x} / r\left(L_{x}\right)$, where $r$ is the fraction of the latitude circle occupied by the ocean basin. The horizontal dimensions of the ocean basin are approximately the ones of the North Pacific. The ocean consists in a single layer of constant thickness $H$ representing the thermocline waters, which temperature varies according to air-sea heat flux and wind-forced advection. Cartesian geometry is used for simplicity and the equations are projected on a $\beta$-plane centered at $45^{\circ} \mathrm{N}$. As the model geometry, the model equations are identical to those of C00 (except for the wind-stress meridional boundary condition), where they are carefully derived and justified, so we simply give here the minimum details required for understanding the present work complementing $\mathrm{C} 00$. The equations of motion are discretized using regular finite differences on an Arakawa $\mathrm{C}$ grid, and Euler forward (not centered and first order) time-stepping is used.

[Figure 1 about here.]

\subsection{The diagnostic atmosphere model}

On climatological time scales (interannual to interdecadal), due to its low thermal capacity and fast adjustment time, the atmosphere can be considered in thermal equilibrium (i.e. the atmosphere is assumed to adjust instantaneously to the ocean state). Therefore we consider that our zonally-averaged one-layer atmosphere is in energy balance between the meridional turbulent heat transport divergence, the incoming short-wave solar radiation $Q_{i}(y)$ at the top of the atmosphere, the re-emitted long-wave flux according to the linearized Stefan-Boltzmann's law $A+B \theta_{s}$ (around the Boussinesq temperature $\Theta$ ), and the zonallyaveraged air-sea heat flux: $F(y)+\lambda\left(\theta_{s}-\overline{T_{s}}\right)$, where $\theta_{s}$ is the surface air temperature, $\overline{T_{s}}$ the zonally-averaged sea surface temperature $T_{s}$, and $\lambda$ the bulk transfer coefficient. The meridional profiles of $Q_{i}(y)$ and $F(y)$ are shown in C00. A picture of the meridional plane model, showing the heat flux at the top of the atmosphere and at the ocean surface, is given in Fig. 2. The only external forcing is the prescribed incoming solar radiation at the top of the atmosphere $Q_{i}(y)$ and at the ocean surface $F(y)$, which meridional profiles are 
shown in C00. Thus the heat conservation equation gives:

$$
-C_{p a} \rho_{s} k_{s} d_{e} \partial_{y}^{2} \theta_{s}=Q_{i}(y)-\left(A+B \theta_{s}\right)-r\left[F(y)+\lambda\left(\theta_{s}-\overline{T_{s}}\right)\right]
$$

where $C_{p a}$ is the heat capacity of the atmosphere, $\rho_{s}$ the air density at the sea level, $k_{s}$ is the atmospheric eddy diffusivity and $d_{e}=d D /(d+D)$ is an effective scale with $d(D)$ the vertical scale of the atmospheric eddy diffusivity (density).

[Figure 2 about here.]

Following Green (1970), the zonally-averaged surface wind stress $\tau$ equals the verticallyintegrated divergence of momentum flux, related to the atmospheric potential temperature through the parameterization of eddy heat flux and quasigeostrophic potential vorticity (PV) flux by a turbulent diffusion law. Using a linearized drag law between the surface stress and surface wind, C00 obtains:

$$
\tau-\frac{d_{e} k_{s}}{\gamma} \partial_{y}^{2} \tau=-\frac{\rho_{s} k_{s} d_{e}}{d}\left[\beta d+\frac{f}{S}\left(\partial_{y} \theta_{s}+L_{\rho}^{2} \partial_{y}^{3} \theta_{s}\right)\right],
$$

where $\gamma$ is the surface drag coefficient, $f$ the Coriolis parameter, and $\beta=\partial_{y} f$. $L_{\rho}$ is the first baroclinic deformation radius of the atmosphere given by

$$
L_{\rho}=\left(\frac{d d_{e} g S}{f^{2} \Theta}\right)^{\frac{1}{2}} \sim 650 \mathrm{~km}
$$

where $g$ is the gravitational acceleration. On the left hand side of (2), the first term is the surface wind stress, the second represents the contribution from the relative vorticity gradient associated with the surface wind shear. On the right hand side, the first term is the contribution from the planetary vorticity gradient $(\beta)$, the second results from the contribution of stretching terms gradients of both PV and eddy heat flux, and the third, the contribution of relative vorticity gradient obtained from the integrated thermal wind balance.

C00 used a zero wind stress boundary condition at the meridional boundaries: $\tau=0$ at $y=0, L_{y}$, such that no transport of eddy momentum is allowed through the boundaries. 
Hence, two additional gyres of weak meridional extent are generated close to these boundaries. We found it preferable to choose a free-slip boundary condition: $\partial_{y} \tau=0$ at $y=0$, $L_{y}$, that ensures zero momentum flux and the continuity of eddy momentum transport

$$
\partial_{y} \tau=-\partial_{y}^{2} \int_{0}^{\infty} \rho \overline{u^{\prime} v^{\prime}} d z=0 \text { at } y=0, L_{y} .
$$

We also impose a conservative constraint on the zonal stress such that there is no net transfer of angular momentum:

$$
\int_{0}^{L_{y}} \tau d y=0
$$

that allows us to determine the vertical scale of eddy-diffusivity $d$ by an iterative method. The values of all constants and parameters are given in Table 1.

[Table 1 about here.]

\subsection{The prognostic ocean model}

The ocean model represents a vertically-homogeneous thermocline of constant depth $H$ forced by surface wind and heat flux. The ocean temperature (SST) varies with air-sea fluxes and the oceanic eddies are parameterized throught the turbulent diffusion $K_{h}$, which is isotropic here. The heat balance, a simple advection-diffusion-forcing equation, allows us to calculate the evolution of SST, assuming that the heat transport is only due to the wind-driven circulation (through the streamfunction $\Psi$, expressed in Sverdrups $-1 \mathrm{~Sv}=$ $\left.10^{6} \mathrm{~m}^{3} \mathrm{~s}^{-1}\right)$, i.e. there is no contribution of the thermohaline circulation:

$$
C_{p w} \rho_{w}\left[H \partial_{t} T_{s}-\partial_{x}\left(T_{s} \partial_{y} \Psi\right)+\partial_{y}\left(T_{s} \partial_{x} \Psi\right)\right]=F(y)+\lambda\left(\theta_{s}-T_{s}\right)+C_{p w} \rho_{w} H \nabla \cdot\left(K_{h} \nabla T_{s}\right)
$$

where $C_{p w}$ and $\rho_{w}$ are respectively the heat capacity and the density of seawater. Zero heat flux conditions are applied normal to basin boundaries.

The mechanical balance equation is derived from the large scale limit of the quasigeostrophic reduced-gravity potential vorticity equation for a two-layer ocean model (one at rest below the thermocline at depth $H=1000 \mathrm{~m}$ ) - the nonlinear terms are purposefully 
neglected such that no intrinsic variability like mesoscale eddies can spontaneously appear:

$$
\partial_{t} \Psi-\beta R^{2} \partial_{x} \Psi=R^{2} \rho_{w}^{-1} \partial_{y} \tau+A_{h x} \partial_{x}^{2} \Psi+A_{h y} \partial_{y}^{2} \Psi
$$

where $c=\beta R^{2}$ is the speed of long baroclinic Rossby waves, $R$ the first baroclinic deformation radius ( $35 \mathrm{~km}$ here), $A_{h x}$ and $A_{h y}$ respectively the eddy diffusivity in the zonal and meridional direction. No-normal-flow lateral boundary conditions imply that $\Psi$ is a constant on the boundary, and we simply take $\Psi=0$. Relatively low eddy-diffusivity is used in the ocean $\left(K_{h}=A_{h y}=200 \mathrm{~m}^{2} \mathrm{~s}^{-1}\right)$ for oscillations to be sustained. However a proper representation of the 'Stommel' frictional western boundary layer (thickness $\delta_{S}=A_{h x} / c$ ) requires larger diffusivity in the zonal direction $\left(2000 \mathrm{~m}^{2} \mathrm{~s}^{-1}\right)$. Such values of diffusion are not in contradiction with the observations from lagrangian floats, which show a clear anisotropy of the eddy-diffusivity (Ollitrault and Colin de Verdière, 2002).

\subsection{Wind stress response to temperature anomalies}

To understand the response of the wind stress to perturbation in the atmospheric temperature (therefore oceanic), we carry out a simple scaling of the momentum balance (2). From the left-hand side, a critical meridional scale $L_{d}$ emerges for measuring the relative importance of the contribution of relative vorticity gradient associated with the surface wind shear compared to the wind stress:

$$
L_{d}=\left(\frac{d_{e} k_{s}}{\gamma}\right)^{\frac{1}{2}} \sim 550 \mathrm{~km}
$$

Neglecting the relative variations of $d$ (typically a few percent), we can proceed to the scaling of the perturbed momentum balance equation, assuming a characteristic surface atmospheric temperature anomaly of scale $\theta^{\star}$ and meridional extension scale $L^{\star}$, and choosing the characteristic scale for the surface wind stress anomaly from the thermal wind balance $\tau^{\star}=\theta^{\star} \rho_{s} k_{s} d_{e} f_{0} /\left(d S L^{\star}\right)$ : On the left hand side, the first term is of order $\tau^{\star}$, the second $\tau^{\star}\left(L_{d} / L^{\star}\right)^{2}$; On the right hand side, the first term is constant, the second of order $\tau^{\star}$, and the third $\tau^{\star}\left(L_{\rho} / L^{\star}\right)^{2}$. 
Given the similarity in the scales $L_{d}$ and $L_{\rho}$, only two types of balance between the wind stress anomaly and the atmospheric temperature gradient anomaly show up depending on the meridional scale of the anomaly:

- For large scale perturbations $\left(L^{\star} \gg L_{d}\right.$ and $\left.L^{\star} \gg L_{\rho}\right)$, the wind stress anomaly response to temperature anomaly results from the contribution of stretching terms gradients of both PV and heat,

$$
\tau^{\prime} \simeq-\frac{\rho_{s} k_{s} d_{e} f}{d S} \partial_{y} \theta_{s}^{\prime}
$$

- For small scale perturbations $\left(L^{\star} \ll L_{d}\right.$ and $\left.L^{\star} \ll L_{\rho}\right)$, the contributions from relative vorticity gradient dominate the anomalous momentum balance,

$$
\frac{d_{e} k_{s}}{\gamma} \partial_{y}^{2} \tau^{\prime} \simeq \frac{\rho_{s} k_{s} d_{e} f L_{\rho}^{2}}{d S} \partial_{y}^{3} \theta_{s}^{\prime} .
$$

To compare these two regimes, let now assume that the wind stress anomaly and temperature anomaly are harmonic perturbations in latitude $y$, both proportional to $\exp (i l y)$, with $l$ the meridional wavenumber. Equation (9) can then be written:

$$
\tau^{\prime} \simeq \frac{\rho_{s} f L_{\rho}^{2} \gamma}{d S} \partial_{y} \theta_{s}^{\prime}=\frac{\rho_{s} d_{e} \gamma g}{f \Theta} \partial_{y} \theta_{s}^{\prime} .
$$

Compared to the previous large-scale case, commonly understood as equivalent barotropic, we find for the small-scale harmonic perturbation a similar relationship but with the opposite sign coefficient, although the amplitudes are rather close $\left(2.2 \times 10^{4}\right.$ vs. $3.1 \times 10^{4} \mathrm{~kg}$ $\left.\mathrm{s}^{-2} \mathrm{~K}^{-1}\right)$ : This arises from the cancellation between both contributions to the meridional gradient of relative vorticity, from the surface and thermal winds.

\section{The mean state and decadal variability}

In the following experiments, all the parameters are the same as in C00 (experiment \#25) except for the atmospheric turbulent diffusion $k_{s}$ which is taken to be $2 \times 10^{6} \mathrm{~m}^{2} \mathrm{~s}^{-1}$ here (to sustain the oscillatory solution described in section 3.2 , exp. \#1) instead of $2.7 \times 10^{6}$ 
$\mathrm{m}^{2} \mathrm{~s}^{-1}$ (exp. \#27, leading to a steady state with our boundary condition). The mean state presented in the following section is averaged over several periods of oscillations. Numerical experiments are summarized in Table 2.

[Table 2 about here.]

\subsection{The mean state}

The mean state of the ocean and the atmosphere are displayed in Figs. 3 and 4. In spite of the simplicity and crudeness of the model, the wind structure is relatively well represented with trade winds in the tropics, westerlies in the mid-latitudes and easterlies poleward of $60^{\circ} \mathrm{N}$. Note that the new boundary condition on wind stress produces two ocean gyres instead of four in the case of $\mathrm{C} 00$, as expected. The result of advection by these two gyres is the formation of a strong thermal front at the intergyre boundary around $40^{\circ} \mathrm{N}$. The subpolar gyre is very vigorous, reaching $50 \mathrm{~Sv}$, since the wind stress curl is strong in this region, while the intensity of the subtropical gyre peaks at $25 \mathrm{~Sv}$ around $30^{\circ} \mathrm{N}$. In the latter, warm water is advected by the western boundary current northward, then injected eastward into the ocean interior south of the intergyre boundary. The atmospheric potential temperature extends from $-20^{\circ} \mathrm{C}$ at the pole to $40^{\circ} \mathrm{C}$ at the equator. The northward heat transport by the western boundary current results in an important heat loss within the northwest quadrant of the subtropical gyre $O\left(300 \mathrm{~W} \mathrm{~m}^{-2}\right)$. The intergyre boundary is shifted northward because of the too large poleward domain extension. The overestimation of SST and heat flux in the subpolar gyre results from several factors: the Cartesian geometry responsible for the too large zonal extent of the domain in high latitudes, the absence of sea ice, convection and the thermohaline component of the circulation...

[Figure 3 about here.]

[Figure 4 about here.] 


\subsection{The decadal variability}

After the spin-up (70 yr), the system equilibrates into periodic oscillations with period of $18.2 \mathrm{yr}$ in all fields. The variability is confined south of the intergyre boundary, in the north of the subtropical gyre. Evolution of transport streamfunction anomalies contours superimposed on SST anomalies during one period of the cycle are shown in Fig. 5. The SST and streamfunction anomalies appear as zonal bands in shape, alternatively positive and negative: Anomalies reach $\pm 10^{\circ} \mathrm{C}$ in SST and $\pm 10 \mathrm{~Sv}$ in streamfunction, and their meridional scale is roughly $400 \mathrm{~km}$. SST anomalies formed in the northwest of the subtrop-

ical gyre around $40^{\circ} \mathrm{N}$ propagate southward with a velocity around $2.6 \times 10^{-3} \mathrm{~m} \mathrm{~s}^{-1}$, and clockwise; About 30 yr after their formation the SST anomalies vanish near the western boundary at about $25^{\circ} \mathrm{N}$.

[Figure 5 about here.]

The different terms (anomalies) in the atmospheric momentum equation, diagnosed at year 0 of the cycle, are displayed in Fig. 6a. At first order, the balance of these terms is between the contribution of relative vorticity gradient related to the surface wind stress anomaly and to the thermal wind anomaly - note that during the whole cycle, this balance is well verified. The dimensional analysis in section 2.3 suggests that the model is in the regime of the small meridional scale anomalies.

[Figure 6 about here.]

In response to the formation of SST anomaly in the northwest of the subtropical gyre, the perturbed atmosphere redistributes the anomalous heat flux over the whole width of the domain and, in addition with the oceanic advection, the SST anomaly rapidly extends eastward. The atmospheric temperature gradients, induced by the air-sea heat flux, modify the wind stress and thus perturb the ocean circulation. Figure $6 \mathrm{~b}$ shows that for each SST anomaly, there is a corresponding transport streamfunction anomaly of opposite sign, slightly shifted northward. 
The two advective terms that dominate the oceanic heat balance are the zonal advection of mean temperature by the current anomaly $u^{\prime} \overline{\partial_{x} T_{s}}$ (the overbar is for the time-mean state and the prime is for the deviation from the mean) and the meridonal advection of the temperature anomaly by the mean current $\bar{V} \partial_{y} T_{s}^{\prime}$. These terms have a significant amplitude only close to the western edge (up to $300 \mathrm{~km}$ ). We can now describe more precisely the oscillation mechanism.

Let suppose that, following a northward shift of the intergyre boundary associated with an anomalously strong subtropical gyre, a positive SST anomaly emerges at year 0 in the western boundary current (WBC) around $42^{\circ} \mathrm{N}$ (Fig. 5a): It is reinforced through increased WBC heat transport by the anticyclonic circulation anomaly growing just south of it (the positive SST anomaly is also stretched towards the ocean interior through anomalous eastward advection). As it develops, atmospheric temperatures follow oceanic temperatures through anomalous air-sea heat flux, that induce changes in the winds according to the small scale regime (section 2.3): Anomalous easterlies (westerlies) develop north (south) of the positive SST anomaly, leading to the formation of a cyclonic circulation anomaly just north of it, that already influences the whole width of the basin at year 6 (Fig. 5b). The intergyre boundary is thus moved southward, the WBC weakened, and a negative temperature anomaly emerges at $42^{\circ} \mathrm{N}$ in the WBC (Fig. 5c). Then the cold SST anomaly generates within a few years an anticyclonic streamfunction anomaly which acts to enhance the WBC, a positive SST anomaly reappears, and the cycle repeats itself. A schematic picture of this oscillation mechanism is shown in Fig. 7. During the southward displacement of the SST anomalies, dissipation acts against the reinforcement: At about $35^{\circ} \mathrm{N}$, the meridional gradient of the SST anomalies vanishes, thus the small scale response of the wind stress, and the reinforcement by the streamfunction anomalies weakens. The SST anomalies zonal extension is reduced by the gyre westward currents, and the anomalies are squeezed against the western boundary where they finally disappear.

[Figure 7 about here.] 
Why is the whole anomalies pattern propagating southward away from the intergyre boundary? What is the mechanism of the ocean circulation adjustment to changes in the wind-stress curl? We will see later that the solution of (6) comprises three wave terms, a directly forced wave which is only latitude-dependent and two free waves (one propagating eastward and confined near the western edge, and the other one propagating westward in the interior). The three waves are equal in amplitude because of the boundary condition, and their combination possesses an apparent phase velocity faster than the long wave speed $c=\beta R^{2}$ in a finite width basin (White, 1977; LaCasce, 2000). The zonal geometry structure of the streamfunction anomalies would rather be due to the forced wave which changes the circulation everywhere simultaneously. Nevertheless the signature of the free baroclinic Rossby waves is hidden behind the forced wave, and one can wonder what importance the free waves have in the mechanism of the variability. This is why we have described the oscillation mechanism without taking into account the westward propagation of baroclinic Rossby waves, which is never obvious in our simulations.

\subsection{The role of interactive wind stress and surface heat flux}

For a better understanding of the influence of interactive wind stress and surface heat flux on the variability, we conducted two additional experiments in which the ocean is forced with a steady wind stress or/and a steady surface heat flux (Fig. 8). These forcings were diagnosed from time-averaging the interactive fluxes over several oscillation periods. Then the model is integrated during $200 \mathrm{yr}$ starting from the time-mean state and keeping one or both forcing fields constant.

[Figure 8 about here.]

Note that the kinetic energy of the mean state $\left(332 \mathrm{~J} \mathrm{~m}^{-2}\right)$ is lower than the mean kinetic energy of the oscillations (413 $\mathrm{J} \mathrm{m}^{-2}$ ), meaning that the perturbations which develop during the oscillations contain a significant amount of kinetic energy. With steady wind stress but interactive heat flux, no oscillation appears, while with steady heat flux but 
interactive wind-stress, the model develops large unstable oscillations, while the kinetic energy increases rapidly. This suggests that the source of the energy sustaining the oscillation is the wind stress coupling, while the heat flux has a damping role. Hence, we will analyze further this "amplification" coefficient $a=\rho_{s} g d_{e} \gamma /(f \Theta)$ linking the wind stress anomaly to the atmospheric temperature gradient anomaly for small-scale perturbations (section 2.3).

\subsection{The ocean adjustment through forced and free Rossby waves}

To justify that the circulation variability is due to the directly forced waves rather than the free propagating Rossby waves, we solve following LaCasce (2000) the linear quasigeostrophic reduced-gravity potential vorticity equation, unidimensional in the zonal direction:

$$
\partial_{t} \Psi-c \partial_{x} \Psi=R^{2} \rho_{w}^{-1} \partial_{y} \tau+A_{h x} \partial_{x}^{2} \Psi
$$

with the boundary condition $\Psi=0$ at the western and eastern edges, respectively $x=0, L_{x}$. Note that the mass conserving boundary condition, allowing time dependant boundary value for $\Psi$, may be more appropriate, and have a significant impact on the relative amplitudes of forced and free waves (LaCasce, 2000), but this would not be consistent with the numerical model.

Assuming a wind stress forcing function of time only (no latitudinal or longitudinal variation):

$$
R^{2} \rho_{w}^{-1} \partial_{y} \tau=F \exp (-i \omega t)
$$

the solution to (11) reads:

$$
\begin{aligned}
\Psi(x, t)=\frac{F \exp (-i \omega t)}{i \omega}\left[-1+\exp \left(-\alpha\left(x-L_{x}\right)\right) \frac{\sinh (A x)}{\sinh \left(A L_{x}\right)}\right. & \\
& \left.\quad-\exp (-\alpha x) \frac{\sinh \left(A\left(x-L_{x}\right)\right)}{\sinh \left(A L_{x}\right)}\right],
\end{aligned}
$$

with

$$
\alpha=\frac{c}{2 A_{h x}} ; A=\frac{\sqrt{c^{2}-4 i A_{h x} \omega}}{2 A_{h x}} .
$$


This solution has three components: a directly forced wave only time-dependent, a westward propagating wave required to satisfy the eastern boundary condition, and an eastward propagating wave required to satisfy the western boundary condition. The three waves are thus equal in amplitude due to the boundary conditions. The SST anomalies in the global two-dimensional coupled model are mainly due to the variability of the western boundary current, therefore we are interested in the relative influence of the propagating waves compared to the directly forced wave close to the western boundary.

For the observed period and the model zonal momentum diffusivity, the amplitude of the forced wave is more than twice larger than the eastward and westward propagating waves in the middle of the western boundary current. The temporal correlation between the direcly forced wave and the global solution (13) is maximum in the western half of the basin, while the covariance of the forced and global solution is twice larger than the others in the western boundary current (not shown). Hence, we can make the symplifying assumption that the free Rossby waves are not essential to the western boundary current variability, therefore the temperature variability, in our configuration. Note that the phase difference between the wind-stress and the directly forced wave is a quarter of a period because of the time derivative. The instability should arise from local adjustment of the oceanic flow to changing winds.

\section{A simplified zonally-averaged analytical model}

The zonality of the geometry of the streamfunction and SST anomalies leads us to build a very simple zonally-averaged analytical model derived from the equations of the global model, in order to understand the physical mechanisms governing the scale selection, growth rate and southward propagation of anomalies. It thus appears that the mean surface current is not the only process responsible for the advection of SST anomalies southward. 


\subsection{The zonally-averaged formulation}

For this purpose, we assume that all the anomalies $\left(T_{s}^{\prime}, \Psi^{\prime}, \theta_{s}^{\prime}, \tau^{\prime}\right)$ are zonal and have the same meridional structure as harmonic perturbations $\exp [i(l y-\omega t)]$, where $l>0$ is the meridional wavenumber $\left(l=2 \pi / \sigma\right.$, with $\sigma$ the wavelength) and $\omega=\omega_{r}+i \omega_{i}$ the wave frequency. The solution has a positive (negative) growth rate if $\omega_{i}>0(<0)$, and propagates northward (southward) if $\omega_{r}>0(<0)$.

A linear relation between the surface air temperature anomaly $\theta_{s}^{\prime}$ and the zonallyaveraged oceanic temperature anomaly $T_{s}^{\prime}$ is simply derived from the heat balance in the atmosphere (1):

$$
\theta_{s}^{\prime}=\frac{r \lambda}{C_{p a} \rho_{s} k_{s} d_{e} l^{2}+B+r \lambda} \overline{T_{s}^{\prime}}=\delta(l) \overline{T_{s}^{\prime}} .
$$

From the momentum balance (2), where we neglect the relative variations of the vertical scale of the atmospheric eddy diffusivity $d$ (its time average and standard deviation in the reference simulation are respectively 6536 and $4 \mathrm{~m}$ ), we obtain the relation between the wind stress anomaly $\tau^{\prime}$ and the atmospheric temperature anomaly $\theta_{s}^{\prime}$ :

$$
\tau^{\prime}=i \alpha(l) \overline{T_{s}^{\prime}}, \quad \text { where } \quad \alpha(l)=-\frac{\rho_{s} k_{s} d_{e} f}{d S} l \delta(l)\left(\frac{1-l^{2} L_{\rho}^{2}}{1+l^{2} L_{d}^{2}}\right)
$$

The proportionality factor $\alpha(l)$ is shown in Fig. 9: The wind stress response is maximum for a wavelength around $1700 \mathrm{~km}$, for which according to (15) the atmospheric temperature anomaly is about 20 times smaller than the SST anomaly (in good agreement with the temperature anomalies profiles $\theta_{s}^{\prime}$ and $T_{s}^{\prime}$ in Fig. $\left.6 \mathrm{~b}\right)$.

[Figure 9 about here.]

The zonal mean barotropic vorticity equation reads:

$$
\partial_{t} \overline{\Psi^{\prime}}=R^{2} \rho_{w}^{-1} \partial_{y} \tau^{\prime}+A_{h y} \partial_{y}^{2} \overline{\Psi^{\prime}}+A_{h x}\left[\partial_{x} \Psi^{\prime}\right]_{0}^{L_{x}}
$$

The term representing the westward propagation of baroclinic Rossby waves only appears implicitly in this equation, through the last boundary term: We have no simple closure for it as a function of zonally-averaged quantities. Without a scaling argument to neglect it, we 
estimate its contribution to the balance in the numerical coupled model: it is less than $7 \%$ of the tendency term in root mean square. Furthermore, the spatio-temporal correlation between both sides of this equation, excluding this last term in the right-hand-side, is $96 \%$. Thus we carry on with this equation without the last term as a good approximation for the zonally-averaged model.

The zonally-averaged ocean heat balance is the most difficult to simplify in terms of zonally-averaged quantities. We have seen that the dominant advective terms are $-\partial_{y} \Psi^{\prime} \partial_{x} T_{s}$ and $\partial_{x} \Psi \partial_{y} T_{s}^{\prime}$. We approximate the first term, zonally-averaged, by $-\partial_{y} \overline{\Psi^{\prime}} \times$ $\overline{\partial_{x} T_{s}}$, since their relative root mean square difference is less than $11 \%$, and the spatiotemporal correlation between both terms is $98 \%$. In order to take into account the mean southward current in the ocean interior east of the western boundary current, we approximate the second term by $\overline{\partial_{x} \Psi_{\text {int }}} \times \partial_{y} \overline{T_{s}^{\prime}}$, where $\overline{\partial_{x} \Psi_{\text {int }}}$ is proportional to the mean interior (southward) meridional velocity $\overline{V_{i n t}}$ : Note that this approximation is not satisfying, since the spatio-temporal correlation between both terms is only $36 \%$. Thus, the simplified zonally-averaged heat balance equation is written, for the subtropical gyre:

$$
\partial_{t} \overline{T_{s}^{\prime}}-H^{-1} \overline{\partial_{x} T_{s}} \partial_{y} \overline{\Psi^{\prime}}+\overline{V_{i n t}} \partial_{y} \overline{T_{s}^{\prime}}=\frac{\lambda}{C_{p w} \rho_{w} H}\left(\theta^{\prime} s-\overline{T_{s}^{\prime}}\right)+K_{h} \partial_{y}^{2} \overline{T_{s}^{\prime}} .
$$

These 4 coupled equations (15-18) lead to a second-order dispersion relation for $\omega$ :

$$
\omega^{2}+i \omega\left[l^{2}\left(A_{h y}+K_{h}\right)+i l \overline{V_{i n t}}-\zeta\right]+i l^{2} \chi-A_{h y} l^{2}\left(K_{h} l^{2}-\zeta+i l \overline{V_{i n t}}\right)=0,
$$

where

$$
\chi(l)=-\alpha(l) \frac{R^{2} \overline{\partial_{x} T_{s}}}{\rho_{w} H} ; \zeta(l)=\frac{\lambda(\delta(l)-1)}{C_{p w} \rho_{w} H} .
$$

The solution consists of a wave propagating northward, which is always damped, and a wave propagating southward, which has a positive growth rate for wavelength larger than $700 \mathrm{~km}$ : Hence, this is the one that will emerge. Real and imaginary parts, representing respectively the propagation and growth/damping rate, are displayed in Fig. 10 for differents values of $\overline{V_{\text {int }}}$ in the subtropical gyre. In analogy with the global model $\overline{\partial_{x} T_{s}}$ is taken equal to $-2 \times 10^{-6} \mathrm{~K} \mathrm{~m}^{-1}$, its mean value in the subtropical gyre. Figure 10 shows that the southward advection of SST anomalies by the mean current $\overline{V_{\text {int }}}$ does not 
significantly modify the final southward propagation. When the interior mean meridional velocity $\overline{V_{i n t}}$ varies from -2 to $0 \mathrm{~mm} \mathrm{~s}^{-1}$, the period and the growth rate only slightly increase. This suggests that the southward propagation of SST anomalies is hardly due to the advection by the mean oceanic current, but predominantly to a prevailing mechanism of self-advecting ocean-atmosphere coupled waves, where anomalies of temperature and ocean streamfunction are out of phase in latitude, as sketched in Fig. 7.

[Figure 10 about here.]

The analytical expressions for the pulsation $\omega_{r}$ and the growth rate $\omega_{i}$ are given in the simple case where $\overline{V_{\text {int }}}=0$ and $K_{h}=A_{h y}=D_{h}$ (as in C00). For $\overline{\partial_{x} T_{s}}<0$, the two solutions are simplified in the approximation $4 l^{2} \chi / \zeta^{2} \gg 1$ (well-verified for wavelength shorter than $2500 \mathrm{~km}$ ), which means that the adjustment time of the circulation to wind stress anomalies is shorter than the adjustment time of SST to thermal forcing anomalies:

$$
\omega_{r}(l)=\mp l \sqrt{\frac{\chi(l)}{2}} ; \omega_{i}(l) \sim-l^{2} D_{h} \pm l \sqrt{\frac{\chi(l)}{2}} .
$$

In a linear framework, we expect the meridional scale of the anomalies that develop to be the wavelength with the maximum growth rate, that is $1300 \mathrm{~km}$ here, which is close to the observed scale of the anomalies in the nonlinear coupled model $(1200 \mathrm{~km})$ :

The southward phase velocity is then $\sqrt{\chi / 2} \sim 2.3 \mathrm{~mm} \mathrm{~s}^{-1}$ for $\overline{\partial_{x} T_{s}}=-2 \times 10^{-6} \mathrm{~K} \mathrm{~m}^{-1}$, as compared to about $2.6 \mathrm{~mm} \mathrm{~s}^{-1}$ in the numerical model. Equation (20) also suggests that the growth rate is stronger south of the intergyre boundary than north of it, since in the coupled model $\overline{\partial_{x} T_{s}}$ is stronger in the subtropical gyre than in the subpolar gyre (where temperature is more uniform because of its stronger intensity). This could explain why the variability mainly affects the subtropical rather than the subpolar gyre.

\subsection{A simple wave equation}

We try further to derive a simple wave equation that would account for the amplification and southward propagation of the SST anomalies. We need to make several more assumptions in order to simplify the set of equations (15-18): We first eliminate the diffusivity 
terms in both (17) and (18), that are not essential for the growth and southward propagation of the anomalies, but only affect the scale selection corresponding to the strongest growth rate. For wavelength smaller than $2 \pi \sqrt{C_{p a} \rho_{s} k_{s} d_{e} /(B+r \lambda)}(\sim 6000 \mathrm{~km})$, the atmospheric heat balance equation becomes:

$$
\partial_{y}^{2} \theta_{s}^{\prime}=-\frac{r \lambda}{C_{p a} \rho_{s} k_{s} d_{e}} \overline{T_{s}^{\prime}}
$$

The small scale balance in the momentum equation (9) reads, after developing $L_{\rho}$ :

$$
\partial_{y}^{2} \tau^{\prime}=\frac{\rho_{s} d_{e} \gamma g}{f \Theta} \partial_{y}^{3} \theta_{s}^{\prime}
$$

After removal of the viscosity terms, the zonal mean barotropic vorticity equation (17) reduces to the forced wave solution:

$$
\partial_{t} \overline{\Psi^{\prime}}=R^{2} \rho_{w}^{-1} \partial_{y} \tau^{\prime}
$$

We have shown that the advection of SST anomalies by the mean interior meridional velocity $\overline{V_{i n t}}$ is not crucial for the positive growth rate and southward propagation: we thus remove it from the heat balance equation. Then, without the damping and diffusive terms, the zonally-averaged oceanic heat balance (18) reads, within the subtropical gyre:

$$
\partial_{t} \overline{T_{s}^{\prime}}=-\frac{\left|\overline{\partial_{x} T_{s}}\right|}{H} \partial_{y} \overline{\Psi^{\prime}}
$$

where $\overline{\partial_{x} T_{s}}$ is the mean zonal gradient of SST in the subtropical gyre $(<0)$. This new set of equations $(22-25)$ leads to a single wave-like equation for $\overline{T_{s}^{\prime}}$ :

$$
\partial_{t}^{2} \overline{T_{s}^{\prime}}-\eta^{2} \partial_{y} \overline{T_{s}^{\prime}}=0 ; \eta^{2}=\frac{R^{2}\left|\overline{\partial_{x} T_{s}}\right| g \gamma r \lambda}{\rho_{w} H C_{p a} k_{s} f \Theta} .
$$

Seeking solutions of the form $\exp [i(l y-\omega t)]$ leads to the dispersion relation: $\omega^{2}=-i l \eta^{2}$, wich gives two solutions:

- $\omega=(1-i) \eta \sqrt{l / 2}$ : a damped solution propagating northward;

- $\omega=(i-1) \eta \sqrt{l / 2}$ : an amplified solution propagating southward. 
We expect only the latter solution to emerge: Note that the phase velocity and the period both increase here with the square root of the wavelength. The meridional scale of the anomalies is given through (15) and (16) (see Fig. 10). With a wavelength of $1300 \mathrm{~km}$ and $\overline{\partial_{x} T_{s}}=-2 \times 10^{-6} \mathrm{~K} \mathrm{~m}^{-1}$, the phase velocity is $2.7 \mathrm{~mm} \mathrm{~s}^{-1}$ and the period is $14.9 \mathrm{yr}$. Hence, given the meridional scale of SST anomalies, (26) provides a valuable estimate of southward phase velocity and period in good agreement with the full coupled model.

\section{Parameter sensitivity analysis}

Having now determined the physical mechanisms responsible for this decadal coupled mode, we try to estimate its robustness to various model paramaters. We begin by comparing the variability found with the boundary condition of zero wind stress (C00, exp. \#2) and the boundary condition of zero wind stress derivative (exp. \#1). Since no major difference arise from applying either condition, we use the boundary condition of zero wind stress to analyze successively the influence of the Coriolis parameter $f$ and the drag coefficient $\gamma$ on the characteristics of the oscillations (period and amplitude): This validates the use of the coefficient $a=\rho_{s} d_{e} \gamma g /(f \Theta)$, linking the wind stress anomaly to the meridional atmospheric temperature gradient anomaly, as the amplification factor for such small meridional scale variability. Then we look at the influence of the dissipative processes, through horizontal temperature and momentum diffusion. Overall, the oscillation period is quite robust to these parameter changes, as summarized in Table 2.

\subsection{Influence of the Coriolis parameter}

We run the model for different values of the Coriolis parameter, ranging from $2 \Omega \sin \left(22^{\circ} \mathrm{N}\right)$ to $2 \Omega \sin \left(67^{\circ} \mathrm{N}\right)$, where $\Omega$ is the Earth rotation rate (exp. \#3-6, note that in our $\beta$-plane formulation, $f$ in the wind-stress equation and $\beta$ in the oceanic Rossby waves speed are

independent). The oscillation period and amplitude decrease when $f$ increases (Fig. 11a), hence the Coriolis parameter has a damping effect on the oscillation. The oscillation 
amplitude even becomes irregular when $f$ is smaller than $2 \Omega \sin \left(33^{\circ} \mathrm{N}\right)$. This is in good agreement with the formula of the amplification factor $a$.

The integration of $(2)$ from equator $(y=0)$ to pole $\left(y=L_{y}\right)$ with the zero-net-stress constraint provides an approximation for the vertical scale of the atmospheric eddy diffusivity:

$$
d \approx \frac{f\left(\theta_{s}(0)-\theta_{s}\left(L_{y}\right)\right)}{\beta S L_{y}}
$$

This approximation is valid everywhere except close to the edges where the first order derivatives terms become significant. This relation shows that $d$ and $f$ act in opposite way on the amplification coefficient $a$, since $d$ increases with $f$. However, the large variations of $d$ with $f$, more than doubling in the explored range of $f$, do not allow to compensate for the direct influence of $f$ on $a$.

\subsection{Influence of the surface drag coefficient}

The wind stress is linearly related to the surface wind through the surface drag coefficient $\gamma$. We run the model for different values of this coefficient, ranging from 0.02 to $0.036 \mathrm{~m}$ $\mathrm{s}^{-1}$ (exp. \#7-10). When the coupling is stronger (large $\gamma$ ), the surface winds are more intense, and the oscillation amplitude increases linearly with $\gamma$ (Fig. 11b): Therefore $\gamma$ acts as an amplifier. The variations of the vertical scale of eddy diffusivity are negligible here $(7230 \pm 30 \mathrm{~m})$. Note that the parameters $f$ and $\gamma$ are the only ones influencing directly the amplification coefficient $a$.

[Figure 11 about here.]

\subsection{Influence of the dissipation and model resolution}

The oceanic horizontal diffusion has a very strong damping influence on the oscillations (exp. \#11-14). Increasing the horizontal diffusion $K_{h}$ smooths the horizontal gradients of temperature and reduces the wind stress response to SST anomalies, hence the oscillations amplitude decreases. Values larger than $800 \mathrm{~m}^{2} \mathrm{~s}^{-1}$ lead to steady-states. 
The oscillation sensitivity to oceanic viscosity in the meridional and zonal direction is even more critical. The influence of the viscosity in the zonal direction on the variability is not obvious to rationalize: When $A_{h x}$ increases from 700 to $2500 \mathrm{~m}^{2} \mathrm{~s}^{-1}$, the oscillation amplitude increases slowly (exp. \#15-18). For values of $A_{h x}$ lower than $700 \mathrm{~m}^{2} \mathrm{~s}^{-1}$, the oscillation amplitude increases strongly with decreasing viscosity. This parameter has little impact on the oscillation period and the structure of SST anomalies remains unchanged.

The model is much more sensitive to the variations of viscosity in the meridional direction $\left(A_{h y}\right)$, that has a strong damping effect on the variability (exp. \#19-22): Not only the amplitude but also the period varies significantly, from $25 \mathrm{yr}$ for $A_{h y}=100 \mathrm{~m}^{2} \mathrm{~s}^{-1}$ to $14 \mathrm{yr}$ for $A_{h y}=300 \mathrm{~m}^{2} \mathrm{~s}^{-1}$. For values of $A_{h y}$ larger than $350 \mathrm{~m}^{2} \mathrm{~s}^{-1}$, the model settles in steady-states, which makes this dissipation coefficient the most critical for the variability. Associated with these changes in meridional viscosity, the variations in the mean southward current within the subtropical gyre are very weak $\left(0.05 \mathrm{~mm} \mathrm{~s}^{-1}\right)$. This supports that the oscillation period depends only weakly on the surface current velocity, but is set through another mechanism, like the self-advecting coupled ocean-atmosphere waves we proposed. Unfortunately, this sensitivity of the period with $A_{h y}$ could not be verified in the analytical model based on harmonic perturbations, since it does not take into account the mean state.

Sensitivity experiments to the atmospheric eddy diffusivity $\left(k_{s}\right)$ have also been performed (exp. \#23-27). Results show similar behavior than C00: that is, oscillation amplitude and period decreases with increasing diffusivity, until steady states are reached.

High resolution experiments $(1000 \times 1000)$ were also carried out to allow low isotropic momentum and temperature horizontal diffusion: These have shown the robustness of the oscillations for $A_{h x}=A_{h y}=K_{h}=200 \mathrm{~m}^{2} \mathrm{~s}^{-1}$ (exp. \#28). Furthermore, this proves that the oscillations are not due to the difference between viscosity in the zonal and meridional directions. However, more realistic oceanic diffusion coefficients $O\left(1000 \mathrm{~m}^{2} \mathrm{~s}^{-1}\right)$ lead to a steady-state: Such a smoothing of the oceanic meridional gradients totally damps potential sources of variability in the model. 


\section{Discussion and conclusion}

We have revisited herein the interdecadal oscillation proposed by Cessi (2000), but for a different lateral boundary condition for the wind stress (free-slip instead of no-slip) that removes spurious ocean gyres close to the equatorward and poleward boundaries. We have found that the oscillatory solution is due to a prevailling ocean-atmosphere coupled mode. The southward propagation of the anomalies results from the relative positions of SST, wind stress and ocean circulation anomalies, rather than the advection by the mean surface current. The role of westward propagating Rossby waves advocated by Cessi (2000) and Gallego and Cessi (2000) is not crucial in our mechanism. Instead the instability arises from the local adjustment of the oceanic flow to changing winds (rather than the delayed, remote mechanism associated with Rossby wave propagation). In a nonlinear quasigeostrophic ocean model coupled to a simple atmosphere, Dewar (2001) shows that the forced response of the inertial recirculation of the gyres also dominates the oceanic -and even climate- variability, rather than baroclinic Rossby waves.

Here, the wind stress coupling appears as the source of energy sustaining the oscillation, while the heat flux has a damping role. The mode shows preferentially a small meridional scale $O(500 \mathrm{~km})$ maximizing the wind stress response to SST anomalies. The positive amplification factor between wind stress anomaly and the atmospheric temperature gradient anomaly reads $a=\rho_{s} g d_{e} \gamma /(f \Theta)$. Note that for large scale perturbations, these two quantities are proportional through a negative factor $a=-\rho_{s} k_{s} d_{e} f /(d S)$. The coupled model exhibits this small scale variability south of the intergyre boundary for both wind-stress boundary conditions $\left(\tau=0\right.$ or $\partial_{y} \tau=0$ at $\left.y=0, L_{y}\right)$, but in a slightly different parameter range, especially in terms of horizontal diffusion.

The zonal shape of the anomalous circulation, and the fact that no Rossby wave propagation has been observed in the fully nonlinear model, suggests that the free Rossby waves are not essential to the interdecadal variability. The following simplified model we proposed uses a zonally-averaged barotropic vorticity equation, which does not represent the propagation of these waves, and yet show an amplification of the SST anomalies with a 
southward propagation.

Solving the linear quasigeostrophic reduced-gravity potential vorticity equation in the zonal direction for our basin width, we have shown that, close to the western boundary, the response of the circulation to changes in surface wind stress is dominated by the forced, only time dependent, Rossby wave. Hence the SST anomalies, generated by low-frequency variability of the western boundary current, may simply respond to these forced Rossby waves.

Consequently, a simple zonally-averaged analytical model is appropriate to understand the origin of the growth rate and propagation of the SST anomalies. It appears that the zonal gradient of mean oceanic temperature averaged within the subtropical gyre is the driving term for the growth of the SST anomalies, while the southward propagation is not only due to the advection by the mean subtropical gyre southward flow but mainly to a prevailing mechanism of self-advecting coupled ocean-atmosphere waves, where temperature and streamfunction anomalies are out of phase in latitude. Also, the scale selection of the most unstable meridional wavelength is obtained at the maximum of the proportionality factor between anomalous surface wind stress and temperature anomaly, i.e. $1300 \mathrm{~km}$ as compared to $1200 \mathrm{~km}$ in the nonlinear model.

Finally, ultimate simplifications have lead to a single wave-like equation for SST anomalies. Given the most unstable meridional wavelength, this single equation provides a southward phase velocity $\left(2.7 \mathrm{~mm} \mathrm{~s}^{-1}\right)$ and a period (14.9 $\mathrm{yr}$ ) in good agreement with the fully coupled model (2.6 $\left.\mathrm{mm} \mathrm{s}^{-1}, 18.2 \mathrm{yr}\right)$.

Gallego and Cessi (2000) already proposed a one-dimensional simplified model for C00 fully coupled model, in which large scale variability emerges: Their anomalies wavelength is about $2500 \mathrm{~km}$ and the period $40 \mathrm{yr}$. The sign of the wind stress curl anomaly $\left(-\partial_{y} \tau^{\prime}\right)$ is opposed to that of the atmospheric temperature anomaly $\left(\theta^{\prime}\right)$, just as for the large scale perturbations regime described in section 2.3. Neglecting the third-order derivative of the atmospheric temperature in the momentum equation and using a linear relaxation law rather than higher-order diffusion for the heat and vorticity flux thus induce fundamental differences between Gallego and Cessi (2000) and Cessi (2000) oscillations, that promote 
the selection of a large-scale instead of a small-scale mode. In fact, we could not obtain any oscillation in our fully-coupled model when the linear relaxation law was used for atmospheric temperature and potential vorticity eddy fluxes, even for smaller ocean viscosities/diffusivities and longer atmospheric relaxation times. Consequently, the existence of oscillations is crucially sensitive to the parameterization of atmospheric eddy fluxes, and given the large uncertainty on these, we cannot conclude on the relevance of the oscillations to the present climate system.

We carried out extensive numerical experiments to test the robustness of the oscillations to several model parameters, with both boundary condition of zero wind stress (C00) or free-slip boundary conditions. Results show the driving role of the surface drag coefficient $\gamma$, and the damping influence of the Coriolis parameter $f$ (in agreement with the expression of the amplification coefficient $a$ ), the horizontal diffusion $K_{h}$, and the viscosity in the meridional direction $A_{h y}$, the latter being the most critical parameter to the oscillation. In fact, oscillations are not sustained for meridional viscosity larger than $350 \mathrm{~m}^{2} \mathrm{~s}^{-1}$, hence stochastic forcing may be necessary to excite them in the real ocean.

Of course there are several deficiencies in our coupled model as compared to the Earth climate system. For instance the oceanic temperature has no influence on the ocean circulation, such that temperature anomalies do not propagate as planetary waves, as in a shallow-water or planetary geostrophic model, but this is not crucial to the oscillation Primeau and Cessi (2001). More crucial is the absence of zonal structure in our atmospheric anomalies that does not allow for coupled ocean-atmosphere perturbations travelling zonally, as discussed by Goodman and Marshall (1999) or Colin de Verdière and Blanc (2001) for instance.

Although such a coupled mechanism of decadal-scale oscillations is an interesting framework to analyze climate variability in data and realistic climate models, such meridional scales of variability do not seem to exist in oceanic or atmospheric observations where larger scales are observed. At the moment, there are no observations of ocean-atmosphere coupled waves that reinforce mutually by propagating southward either, but similar mechanisms may be relevant for large-scale anomalies. 


\section{Acknowledgments}

This work was carried out at the Laboratoire de Physique des Océans (UMR 6523 CNRS IFREMER UBO) in the Université de Bretagne Occidentale, Brest, France. Computational facilities for the high resolution simulations were provided by the Institut du Développement et des Ressources en Informatique Scientifique (IDRIS, Orsay, France). Constructive comments and suggestions from A. Colin de Verdière and two anonymous reviewers are gratefully acknowledged. 


\section{References}

Cessi, P., 2000. Thermal feedback on wind-stress as a contributing cause of climate variability. J. Clim. 13, 232-244.

Colin de Verdière, A., Blanc, M.L., 2001. Thermal resonance of the atmosphere to SST anomalies. Implications for the Antarctic circumpolar wave. Tellus 53A, 403-424.

Dewar, W.K., 2001. On ocean dynamics in midlatitude climate. J. Clim. 14, 4380-4397.

Folland, C.K., Parker, D.E., Kates, F.E., 1984. Worldwide marine temperature fluctuations 1856-1981. Nature 310, 670-673.

Gallego, B., Cessi, P., 2000. Exchange of heat and momentum between the atmosphere and the ocean: a minimal model of decadal oscillations. Clim. Dyn. 16, 479-489.

Ghil, M., Vautard, R., 1991. Interdecadal oscillations and the warming trend in global temperature time series. Nature 350, 324-327.

Goodman, J., Marshall, J., 1999. A model of decadal middle-latitude atmosphere-ocean coupled modes. J. Clim. 12, 621-641.

Green, J.S.A., 1970. Transfer properties of the large-scale eddies and the general circulation of the atmosphere. Q. J. R. Meteorol. Soc. 96, 157-185.

Jin, F.F., 1997. A theory of interdecadal climate variability of the North Pacific oceanatmosphere system. J. Clim. 10, 1821-1835.

LaCasce, J.H., 2000. Baroclinic Rossby waves in a square basin. J. Phys. Oceanogr. 30, 3161-3178.

Latif, M., 1998. Dynamics of interdecadal variability in coupled ocean-atmosphere models. J. Clim. 11, 602-624.

Latif, M., Barnett, T.P., 1994. Causes of decadal climate variability over the north Pacific and north America. Science 266, 634-637. 
—, 1996. Decadal variability over the North Pacific and North America: Dynamics and predictability. J. Clim. 9, 2407-2423.

Levitus, S., 1989. Interpendatal variability of temperature and salinity of intermediate depths of the North Atlantic Ocean, 1970-1974 versus 1955-1959. J. Geophys. Res. 94, 9679-9685.

Ollitrault, M., Colin de Verdière, A., 2002. SOFAR Floats Reveal Midlatitude Intermediate North Atlantic General Circulation. Part II: An Eulerian Statistical View. J. Phys. Oceanogr. 32, 2034-2053.

Primeau, F., Cessi, P., 2001. Coupling between wind-driven currents and midlatitude storm tracks. J. Clim. 14, 1243-1261.

Robertson, A.W., 1996. Interdecadal variability over the North Pacific in a multi-century climate simulation. Clim. Dyn. 12, 227-241.

Sura, P., Lunkeit, F., Fraedrich, K., 1999. Decadal variability in a simplified wind-driven ocean model. J. Phys. Oceanogr. 30, 1917-1930.

Tanimoto, Y., Hanawa, K., Toba, Y., 1993. Characteristic variations of sea surface temperature with multiple time scales in the North Pacific. J. Clim. 6, 1153-1160.

Trenberth, K.E., 1990. Recent observed interdecadal climate changes in the northern hemisphere. Bull. Am. Meteorol. Soc. 71, 988-993.

Venegas, S.A., Mysak, L.A., Straub, D.N., 1998. An interdecadal climate cycle in the South Atlantic and its links to other ocean basins. J. Geophys. Res. 103, $24723-$ 24736 .

Weng, W., Neelin, J.D., 1998. On the role of ocean-atmosphere interaction in midlatitude interdecadal variability. Geophys. Res. Lett. 25, 167-170.

White, W.B., 1977. Annual forcing of baroclinic long waves in the tropical North Pacific. J. Phys. Oceanogr. 7, 50-61. 


\section{List of Figures}

1 Top-view of the coupled mode. The ocean and the atmosphere have the same meridional extension $L_{y}$. The longitudinal extension of the ocean is $L_{x}$, while the one of the atmosphere is $L_{x} / r$, with $r$ the fraction of latitude circle occupied by the ocean basin. . . . . . . . . . . . . . . . . .

2 The meridional-plane model, showing the radiation balance at the top of the atmosphere and the heat flux at the ocean surface. The only external forcing is the prescribed incoming solar radiation at the top of the atmosphere $Q_{i}(y)$ and at the ocean surface $F(y)$. The re-emitted long-wave flux is parameterized according to the linearized Stefan-Boltzmann's law $A+B \theta_{s}$. The sensible heat flux at the air-sea interface is $\lambda\left(\theta_{s}-T_{s}\right) . \ldots . .$.

3 a) The zonally-averaged surface air temperature (dashed) and zonal-mean sea surface temperature. The temperature spreads from $40^{\circ} \mathrm{C}$ at the equator to $-20^{\circ} \mathrm{C}$ at the pole, in the absence of sea ice . b) The net surface heat flux into the ocean $\left(\mathrm{W} \mathrm{m}^{-2}\right)$ varies from $-300 \mathrm{~W} \mathrm{~m}^{-2}$ in the subtropical gyre western boundary current (WBC) to $440 \mathrm{~W} \mathrm{~m}^{-2}$ in the subpolar gyre WBC. The northward heat transport at the intergyre boundary reaches 3

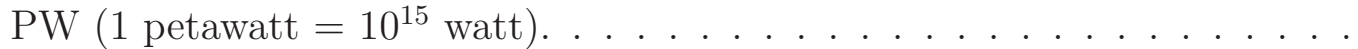

a) The zonally-averaged wind-stress (in $\mathrm{N} \mathrm{m}^{-2}$ ). b) The corresponding transport streamfunction $\Psi$ (thick contours, in $\mathrm{Sv}, 1 \mathrm{~Sv}=10^{6} \mathrm{~m}^{3} \mathrm{~s}^{-1}$ ) superimposed on the oceanic temperature field (thin contours, in ${ }^{\circ} \mathrm{C}$ ). The maximum transport is $50 \mathrm{~Sv}$ (cyclonic) in the subpolar gyre with relatively homogeneous SST, and $25 \mathrm{~Sv}$ (anticyclonic) in the subtropical gyre. The circulation produces a strong thermal front at the intergyre boundary. . . . . . . . . . Anomalies of SST $\left({ }^{\circ} \mathrm{C}\right.$, in color) and transport streamfunction (contours at 0 , $\pm 2, \pm 5, \pm 10 \mathrm{~Sv}$ ) between 24 and $44^{\circ} \mathrm{N}$ every 6 yr over one oscillation period (18.2 yr). The intergyre boundary $(\Psi=0)$ is superimposed (thick solid line). Negative contours are dashed and correspond to cyclonic circulation. SST anomalies spread over $\pm 8^{\circ} \mathrm{C}$, and transport streamfunction anomalies over $\pm 10 \mathrm{~Sv}$. The SST anomalies propagating southward (average velocity around $2.6 \mathrm{~mm} \mathrm{~s}^{-1}$ ) are first reinforced by the circulation anomalies slightly shifted southward, and take about 35 yr to reach the tropics and decay. . . 
6 Atmospheric momentum balance and anomalies in the strong variability region around the intergyre boundary, at year 0 of the oscillation cycle. a) Anomaly terms in the atmospheric momentum equation: surface wind stress $\tau^{\prime}$ (solid), $-\left(\rho_{s} k_{s} d_{e} f /(d S)\right) \partial_{y} \theta^{\prime}$ (dotted), $-\left(d_{e} k_{s} / \gamma\right) \partial_{y}^{2} \tau^{\prime}$ (dashed), and $-\left(\rho_{s} k_{s} d_{e} f L_{\rho}^{2} /(d S)\right) \partial_{y}^{3} \theta^{\prime}$ (dash-dotted). The balance is clearly between the two terms in the meridional gradient of relative vorticity, one related to the surface winds (dashed) and the other to the thermal wind balance (dashdotted), as analysed dimensionaly for small scale perturbations (section 2.3). b) Zonally-averaged anomalies in $\mathrm{SST}$ (solid: $\overline{T_{s}^{\prime}},{ }^{\circ} \mathrm{C}$ ), surface air temperature (dashed: $\theta^{\prime} \times 50,{ }^{\circ} \mathrm{C}$ ), surface wind-stress curl (dash-dotted: $-\partial_{y} \tau^{\prime} \times 10^{8}$, $\mathrm{N} \mathrm{m}^{-3}$ ), and transport streamfunction (dotted: $\overline{\Psi^{\prime}}, \mathrm{Sv}$ ). The air temperature anomalies are 20 times smaller than the SST anomalies. The negative SST anomaly which emerges between 38 and $41^{\circ} \mathrm{N}$ leads to the formation of a positive transport streamfunction anomaly slightly shifted northward. . .

$7 \quad$ Schematic picture of the oscillation mechanism. The left and right panels show respectively a snapshot and the associated trend of the anomalies. SST anomalies in the western boundary current are reinforced by the transport streamfunction anomalies (dashed) located just south (solid arrow). A positive SST anomaly leads to the formation of a negative streamfunction anomaly slightly shifted northward, which slows down (dashed arrow) the western boundary current and induces the following negative SST anomaly. Sensitivity of the model to the wind stress and surface heat flux coupling. With steady surface wind stress (but interactive heat flux) no oscillation appears (straight dotted line), while with steady heat flux (but interactive winds) the model is unstable and large amplitude oscillations arise (dashed line). The solid line is the control experiment. Hence an interactive wind stress is essential to the growth of the SST anomalies and therefore to the variability. . . . . . . . . . . . . . . . . . Influence of SST anomaly wavelength on the amplitude of the wind stress response (assuming harmomic perturbations in latitude). The proportionality factor $\alpha$ between $\tau^{\prime}$ and $\overline{T_{s}^{\prime}}$, defined as $\tau^{\prime}=i \alpha \overline{T_{s}^{\prime}}$, is maximum for a wavelength around $1700 \mathrm{~km}\left(\alpha \sim 5 \times 10^{-3} \mathrm{~N} \mathrm{~m}^{-2} \mathrm{~K}^{-1}\right)$. Note the opposite sign response for the largest wavelength. . . . . . . . . . . . . . . 
10 The wave frequency $\left(\omega=\omega_{r}+i \omega_{i}\right)$ for the solution propagating southward for differents values of the mean meridional velocity $\overline{V_{\text {int }}}$ in the subtropical gyre: $\overline{V_{i n t}}=0 \mathrm{~mm} \mathrm{~s}^{-1}$ (solid line), $\overline{V_{i n t}}=-2 \mathrm{~mm} \mathrm{~s}^{-1}$ (dashed line). The real part of the wave frequency (southward propagation for $\omega_{r}<0$ ) is in thin line, while the corresponding imaginary part (positive growth rate for $\omega_{i}>0$ ) is in thick line. The growth rate is maximum for a wavelength of about 1300 $\mathrm{km}$, to be compared to the actual meridional wavelength of the anomalies in the coupled model, around $1200 \mathrm{~km}$. The solution propagating northward, not shown, has a negative growth rate, hence only the solution propagating southward emerges. In addition, the simplified solutions (21) (dash-dotted) and the wave-like solution of section $4.2(\times)$ are provided: the latter differs at small wavelength since diffusion terms are neglected. . . . . . . . . .

11 The oscillation amplitude as a function of a) the Coriolis parameter, b) the surface drag coefficient (both in log-log plot). The amplitude is measured as the standard deviation of the total oceanic kinetic energy. Crosses are used for unstable oscillations, circles for stable oscillations. . . . . . . . . . 


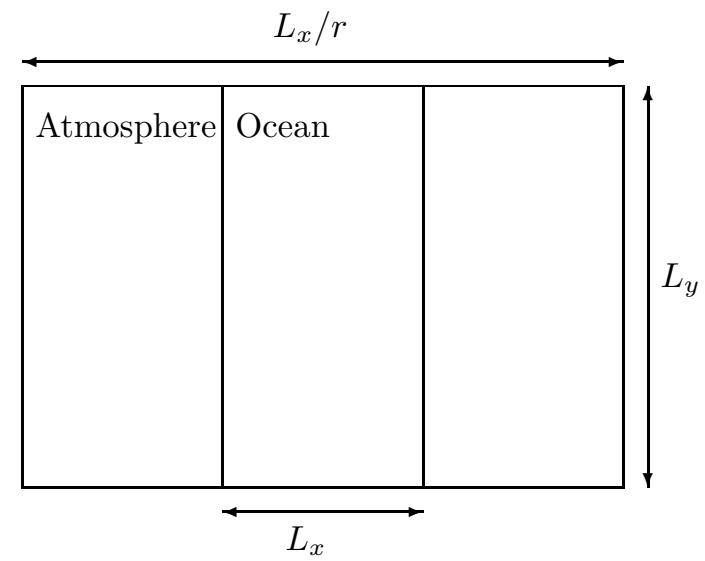

Figure 1: Top-view of the coupled mode. The ocean and the atmosphere have the same meridional extension $L_{y}$. The longitudinal extension of the ocean is $L_{x}$, while the one of the atmosphere is $L_{x} / r$, with $r$ the fraction of latitude circle occupied by the ocean basin. 


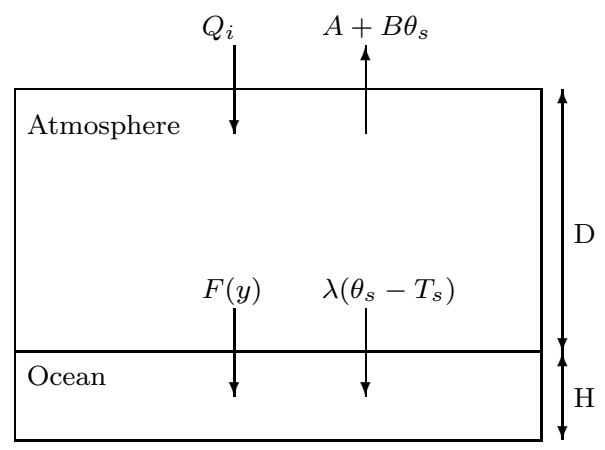

Figure 2: The meridional-plane model, showing the radiation balance at the top of the atmosphere and the heat flux at the ocean surface. The only external forcing is the prescribed incoming solar radiation at the top of the atmosphere $Q_{i}(y)$ and at the ocean surface $F(y)$. The re-emitted long-wave flux is parameterized according to the linearized Stefan-Boltzmann's law $A+B \theta_{s}$. The sensible heat flux at the air-sea interface is $\lambda\left(\theta_{s}-T_{s}\right)$. 
a)

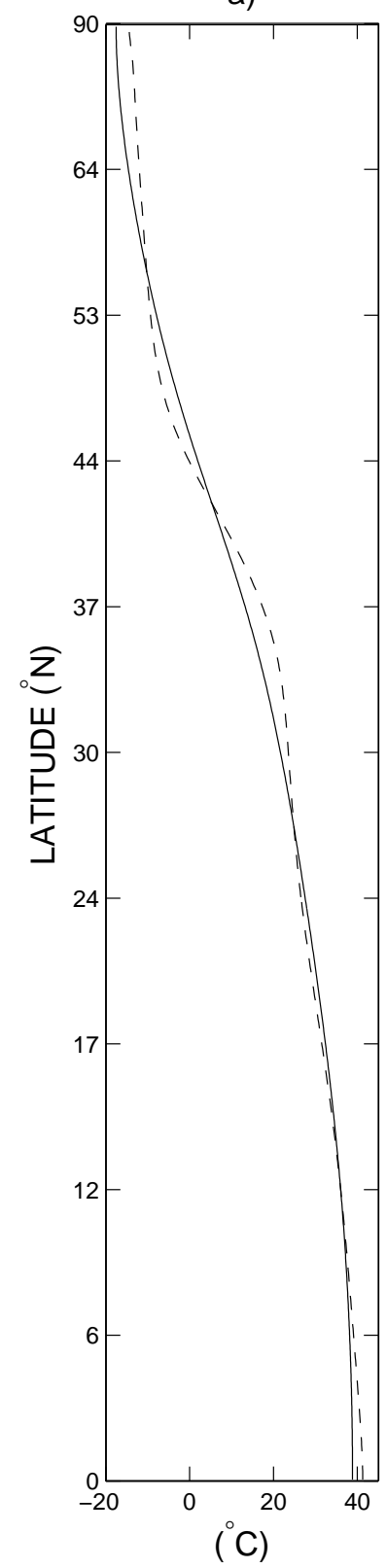

b)

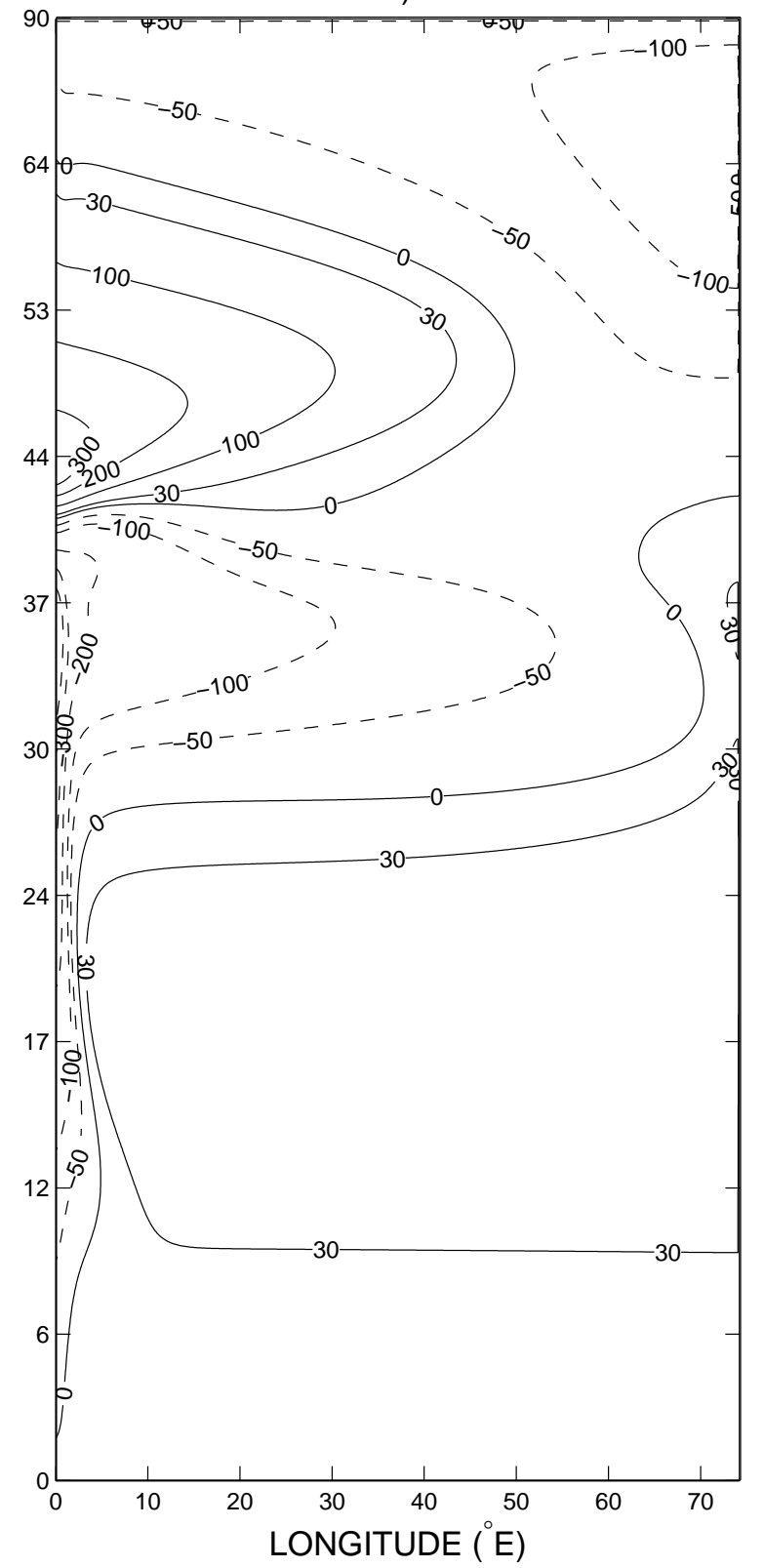

Figure 3: a) The zonally-averaged surface air temperature (dashed) and zonal-mean sea surface temperature. The temperature spreads from $40^{\circ} \mathrm{C}$ at the equator to $-20^{\circ} \mathrm{C}$ at the pole, in the absence of sea ice . b) The net surface heat flux into the ocean $\left(\mathrm{W} \mathrm{m}^{-2}\right)$ varies from $-300 \mathrm{~W} \mathrm{~m}^{-2}$ in the subtropical gyre western boundary current (WBC) to $440 \mathrm{~W}$ $\mathrm{m}^{-2}$ in the subpolar gyre WBC. The northward heat transport at the intergyre boundary reaches $3 \mathrm{PW}$ (1 petawatt $=10^{15}$ watt $)$. 
a)

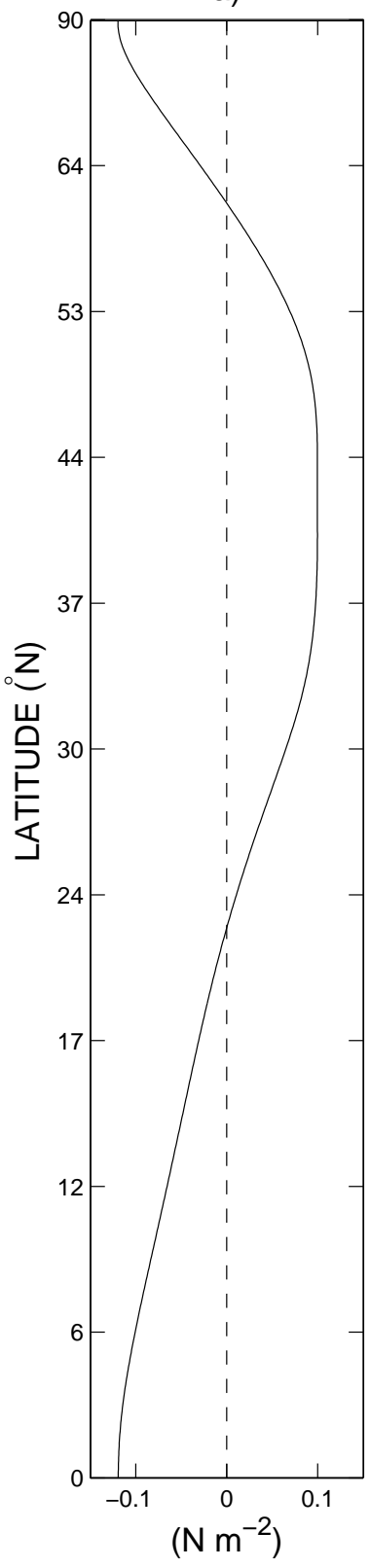

b)

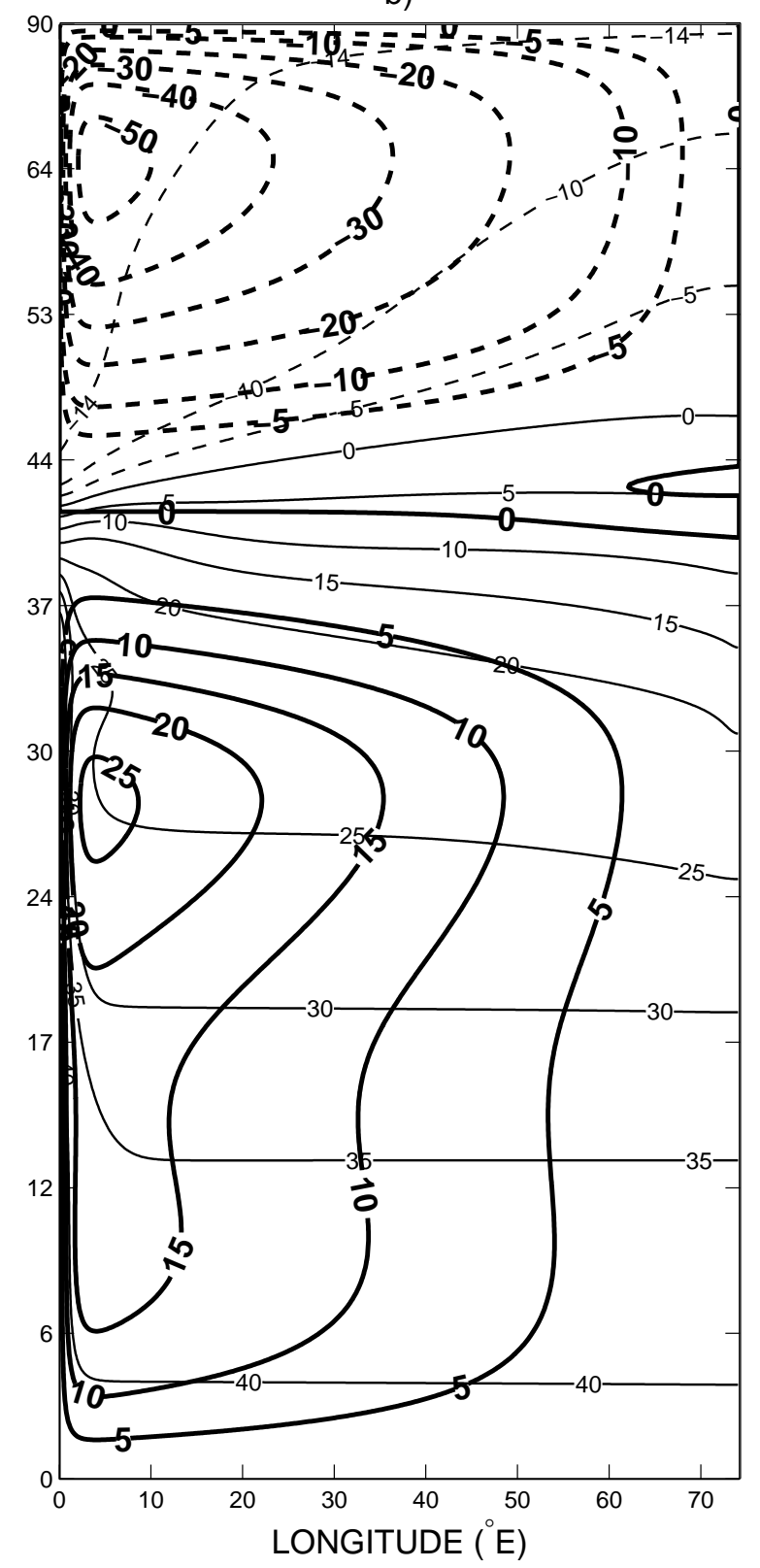

Figure 4: a) The zonally-averaged wind-stress (in $\mathrm{N} \mathrm{m}^{-2}$ ). b) The corresponding transport streamfunction $\Psi$ (thick contours, in Sv, $1 \mathrm{~Sv}=10^{6} \mathrm{~m}^{3} \mathrm{~s}^{-1}$ ) superimposed on the oceanic temperature field (thin contours, in ${ }^{\circ} \mathrm{C}$ ). The maximum transport is $50 \mathrm{~Sv}$ (cyclonic) in the subpolar gyre with relatively homogeneous SST, and $25 \mathrm{~Sv}$ (anticyclonic) in the subtropical gyre. The circulation produces a strong thermal front at the intergyre boundary. 
a) YEARS 0-18
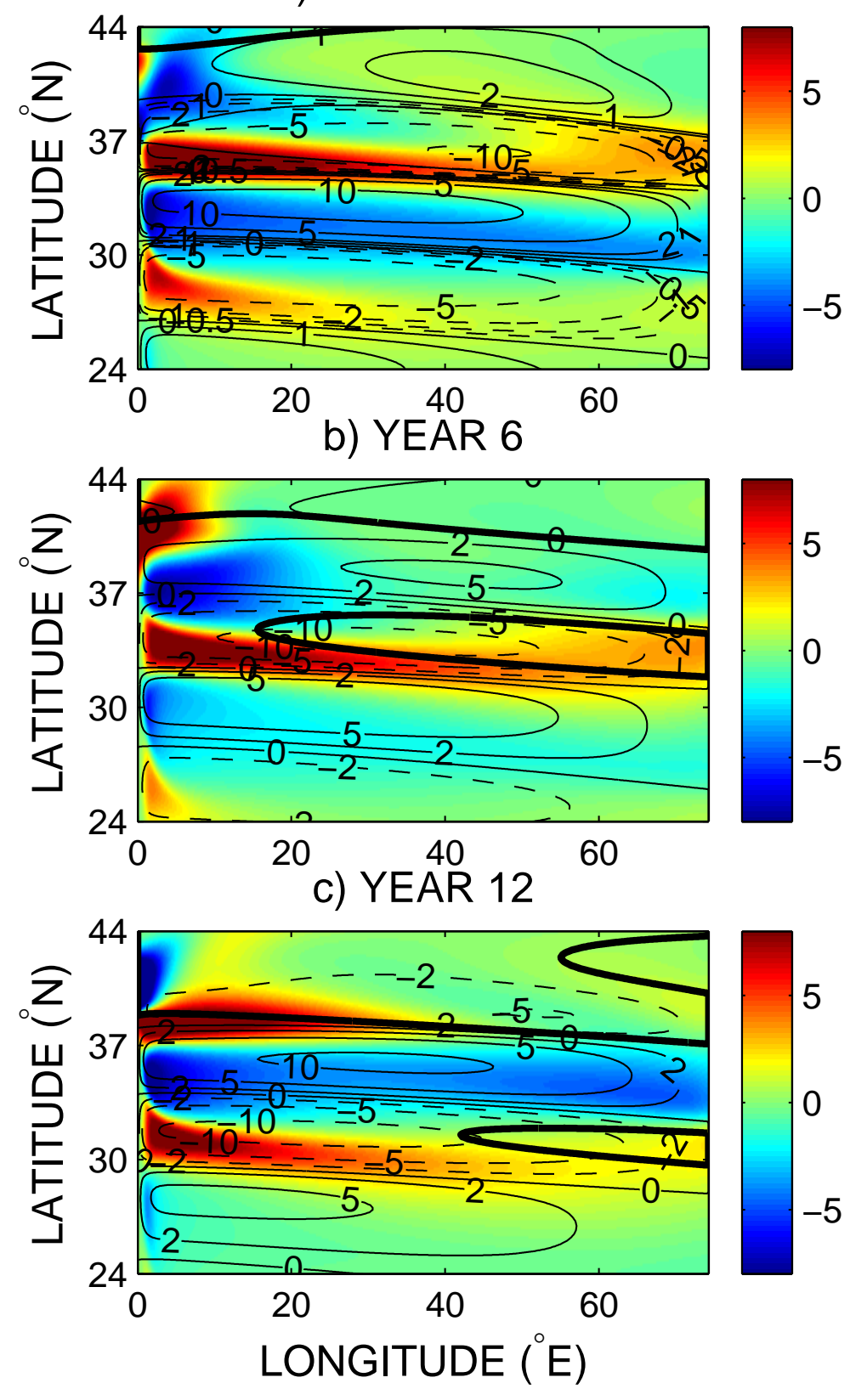

Figure 5: Anomalies of $\operatorname{SST}\left({ }^{\circ} \mathrm{C}\right.$, in color) and transport streamfunction (contours at 0, $\pm 2, \pm 5, \pm 10 \mathrm{~Sv}$ ) between 24 and $44^{\circ} \mathrm{N}$ every 6 yr over one oscillation period (18.2 yr). The intergyre boundary $(\Psi=0)$ is superimposed (thick solid line). Negative contours are dashed and correspond to cyclonic circulation. SST anomalies spread over $\pm 8^{\circ} \mathrm{C}$, and transport streamfunction anomalies over $\pm 10 \mathrm{~Sv}$. The SST anomalies propagating southward (average velocity around $2.6 \mathrm{~mm} \mathrm{~s}^{-1}$ ) are first reinforced by the circulation anomalies slightly shifted southward, and take about $35 \mathrm{yr}$ to reach the tropics and decay. 
a)

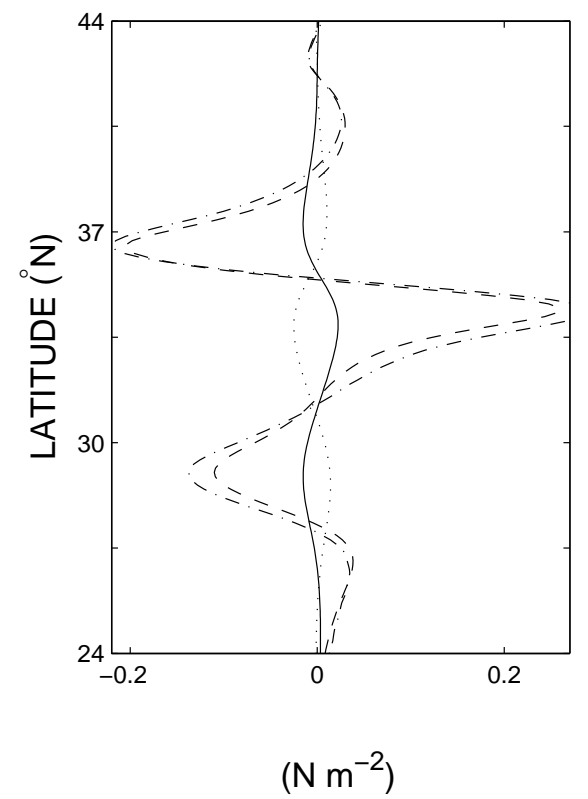

b)

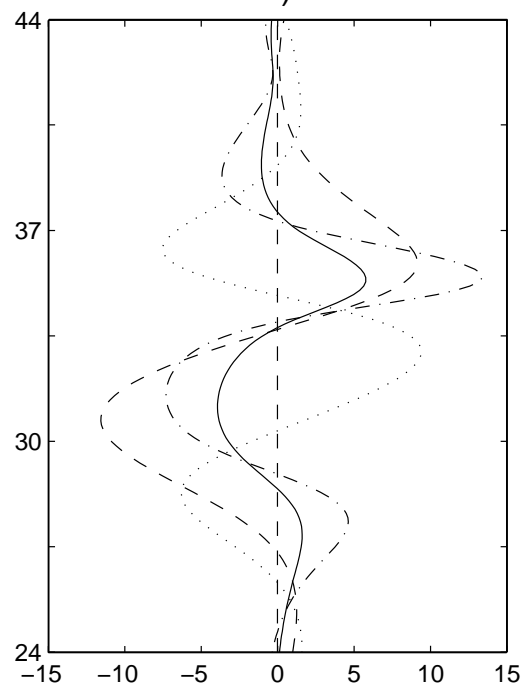

Figure 6: Atmospheric momentum balance and anomalies in the strong variability region around the intergyre boundary, at year 0 of the oscillation cycle. a) Anomaly terms in the atmospheric momentum equation: surface wind stress $\tau^{\prime}$ (solid), $-\left(\rho_{s} k_{s} d_{e} f /(d S)\right) \partial_{y} \theta^{\prime}$ (dotted), $-\left(d_{e} k_{s} / \gamma\right) \partial_{y}^{2} \tau^{\prime}$ (dashed), and $-\left(\rho_{s} k_{s} d_{e} f L_{\rho}^{2} /(d S)\right) \partial_{y}^{3} \theta^{\prime}$ (dash-dotted). The balance is clearly between the two terms in the meridional gradient of relative vorticity, one related to the surface winds (dashed) and the other to the thermal wind balance (dash-dotted), as analysed dimensionaly for small scale perturbations (section 2.3). b) Zonally-averaged anomalies in SST (solid: $\overline{T_{s}^{\prime}},{ }^{\circ} \mathrm{C}$ ), surface air temperature (dashed: $\theta^{\prime} \times 50,{ }^{\circ} \mathrm{C}$ ), surface wind-stress curl (dash-dotted: $-\partial_{y} \tau^{\prime} \times 10^{8}, \mathrm{~N} \mathrm{~m}^{-3}$ ), and transport streamfunction (dotted: $\left.\overline{\Psi^{\prime}}, \mathrm{Sv}\right)$. The air temperature anomalies are 20 times smaller than the SST anomalies. The negative SST anomaly which emerges between 38 and $41^{\circ} \mathrm{N}$ leads to the formation of a positive transport streamfunction anomaly slightly shifted northward. 


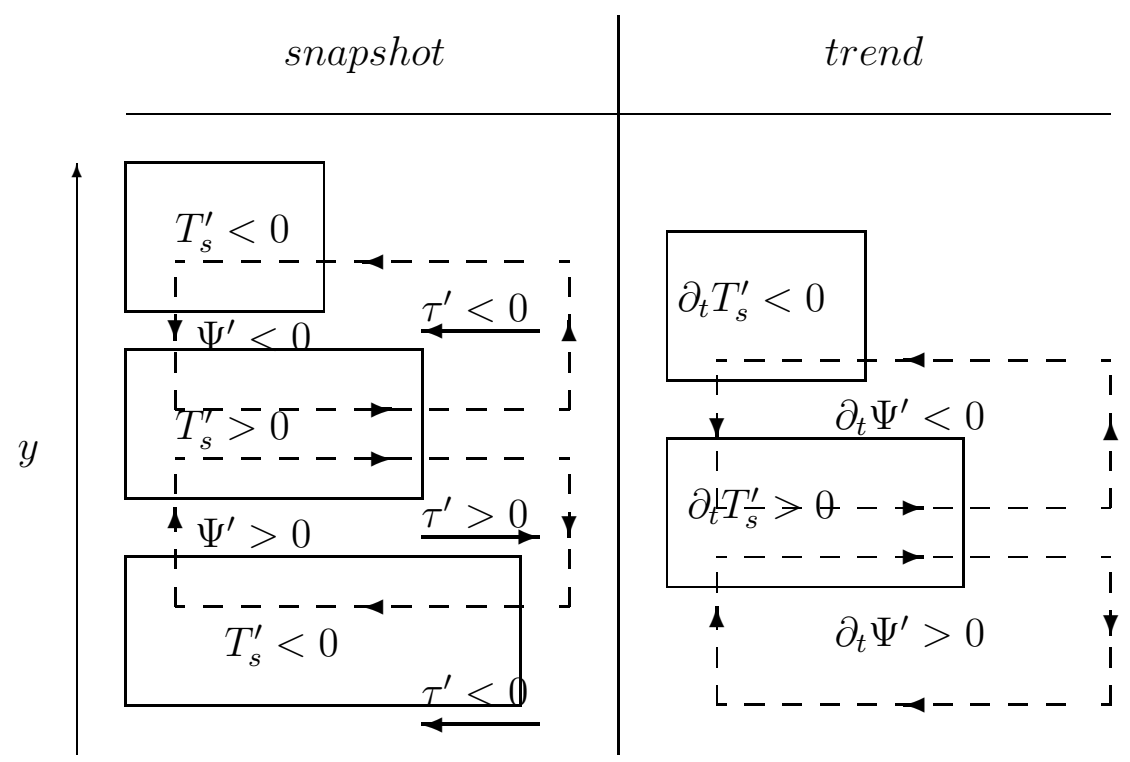

Figure 7: Schematic picture of the oscillation mechanism. The left and right panels show respectively a snapshot and the associated trend of the anomalies. SST anomalies in the western boundary current are reinforced by the transport streamfunction anomalies (dashed) located just south (solid arrow). A positive SST anomaly leads to the formation of a negative streamfunction anomaly slightly shifted northward, which slows down (dashed arrow) the western boundary current and induces the following negative SST anomaly. 


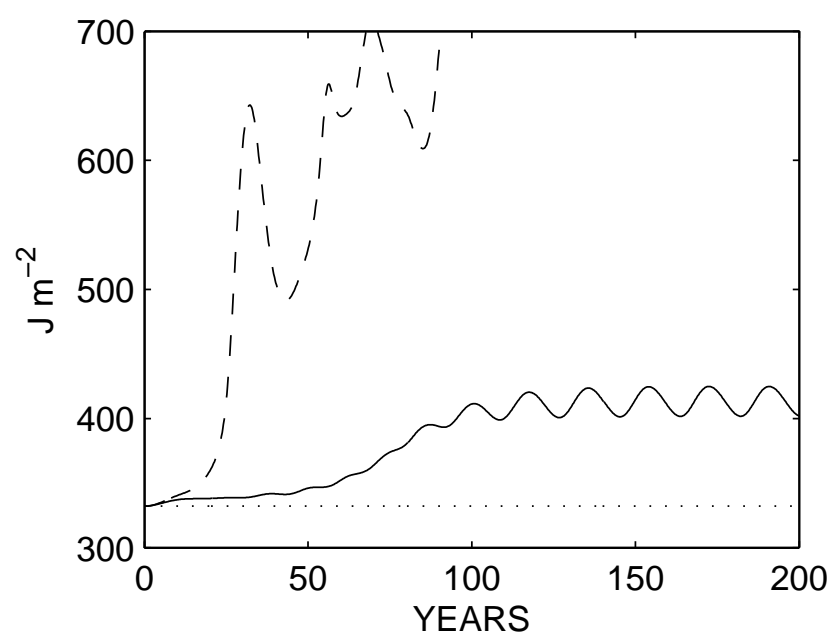

Figure 8: Sensitivity of the model to the wind stress and surface heat flux coupling. With steady surface wind stress (but interactive heat flux) no oscillation appears (straight dotted line), while with steady heat flux (but interactive winds) the model is unstable and large amplitude oscillations arise (dashed line). The solid line is the control experiment. Hence an interactive wind stress is essential to the growth of the SST anomalies and therefore to the variability. 


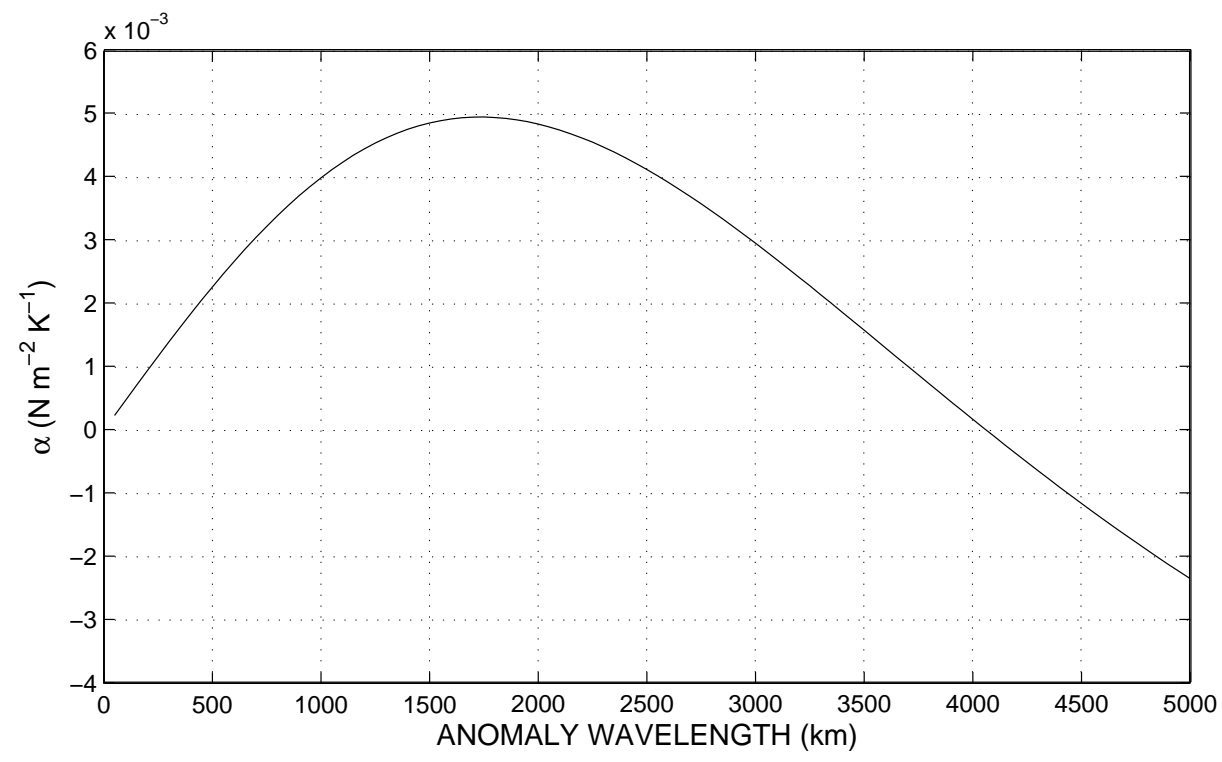

Figure 9: Influence of SST anomaly wavelength on the amplitude of the wind stress response (assuming harmomic perturbations in latitude). The proportionality factor $\alpha$ between $\tau^{\prime}$ and $\overline{T_{s}^{\prime}}$, defined as $\tau^{\prime}=i \alpha \overline{T_{s}^{\prime}}$, is maximum for a wavelength around $1700 \mathrm{~km}\left(\alpha \sim 5 \times 10^{-3}\right.$ $\mathrm{N} \mathrm{m}^{-2} \mathrm{~K}^{-1}$ ). Note the opposite sign response for the largest wavelength. 


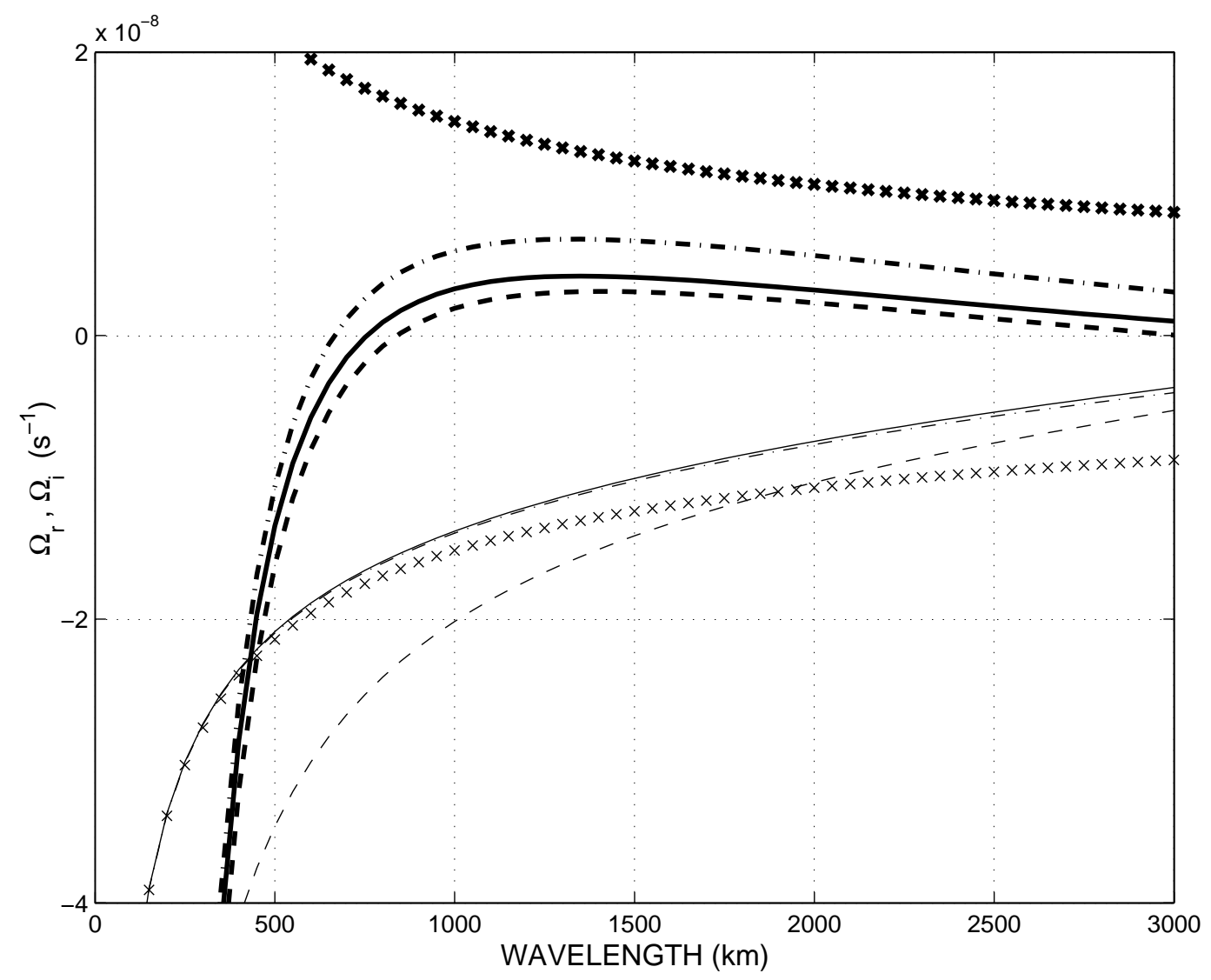

Figure 10: The wave frequency $\left(\omega=\omega_{r}+i \omega_{i}\right)$ for the solution propagating southward for differents values of the mean meridional velocity $\overline{V_{\text {int }}}$ in the subtropical gyre: $\overline{V_{\text {int }}}=0 \mathrm{~mm} \mathrm{~s}^{-1}$ (solid line), $\overline{V_{\text {int }}}=-2 \mathrm{~mm} \mathrm{~s}^{-1}$ (dashed line). The real part of the wave frequency (southward propagation for $\omega_{r}<0$ ) is in thin line, while the corresponding imaginary part (positive growth rate for $\omega_{i}>0$ ) is in thick line. The growth rate is maximum for a wavelength of about $1300 \mathrm{~km}$, to be compared to the actual meridional wavelength of the anomalies in the coupled model, around $1200 \mathrm{~km}$. The solution propagating northward, not shown, has a negative growth rate, hence only the solution propagating southward emerges. In addition, the simplified solutions (21) (dash-dotted) and the wave-like solution of section $4.2(\times)$ are provided: the latter differs at small wavelength since diffusion terms are neglected. 
a)

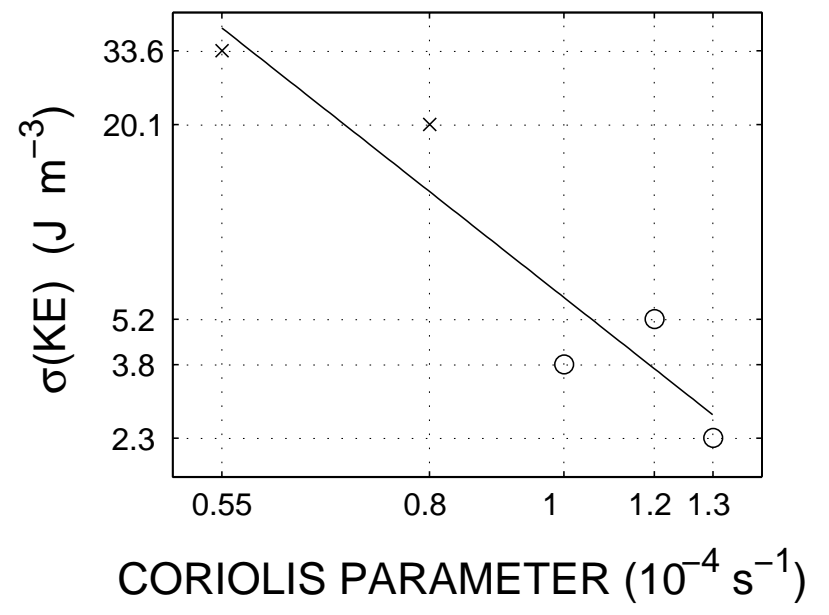

b)

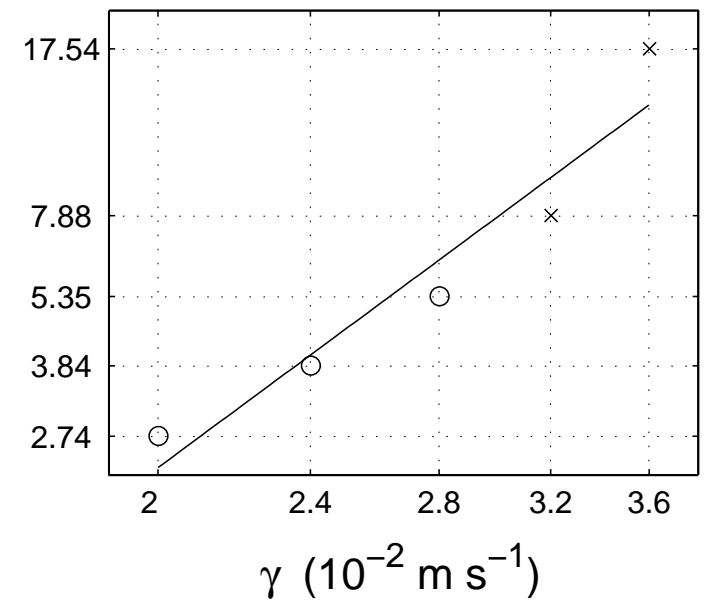

Figure 11: The oscillation amplitude as a function of a) the Coriolis parameter, b) the surface drag coefficient (both in log-log plot). The amplitude is measured as the standard deviation of the total oceanic kinetic energy. Crosses are used for unstable oscillations, circles for stable oscillations. 


\section{List of Tables}

1 Standard values of the model parameters, as in Cessi (2000) except for the

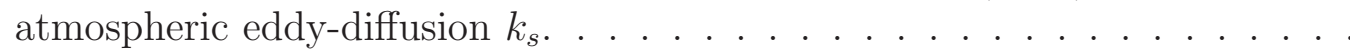

2 Summary of the numerical experiments. BC is the boundary condition for surface wind stress. Oscillation period is provided when available, and oscillation amplitude is measured through standard deviation of oceanic kinetic energy. . . . . . . . . . . . . . . . . . . . . 


\begin{tabular}{|c|c|c|}
\hline & & Atmospheric parameters \\
\hline$D$ & $8000 \mathrm{~m}$ & scale height for reference atmospheric density \\
\hline$\rho_{s}$ & $1.25 \mathrm{~kg} \mathrm{~m}^{-3}$ & reference surface air density \\
\hline$S$ & $5 \times 10^{-3}{ }^{\circ} \mathrm{C} \mathrm{m}^{-1}$ & potential temperature stratification \\
\hline$C_{p a}$ & $1000 \mathrm{~J} \mathrm{~K}^{-1} \mathrm{~kg}^{-1}$ & heat capacity \\
\hline$k_{s}$ & $2 \times 10^{6} \mathrm{~m}^{2} \mathrm{~s}^{-1}$ & eddy diffusivity \\
\hline$f$ & $10^{-4} \mathrm{~s}^{-1}$ & Coriolis parameter at $45^{\circ} \mathrm{N}$ \\
\hline$A$ & $200 \mathrm{~W} \mathrm{~m}^{-2}$ & outgoing longwave parametrization coefficient \\
\hline$B$ & $2.475 \mathrm{~W} \mathrm{~m}^{-2}{ }^{\circ} \mathrm{C}^{-1}$ & $\begin{array}{l}\text { outgoing longwave parametrization coefficient } \\
\text { Ocean parameters }\end{array}$ \\
\hline$L_{x}$ & $8,250 \mathrm{~km}$ & longitudinal extent \\
\hline$L_{y}$ & $10,000 \mathrm{~km}$ & latitudinal extent \\
\hline$H$ & $1000 \mathrm{~m}$ & thermocline depth \\
\hline$\rho_{w}$ & $1000 \mathrm{~kg} \mathrm{~m}^{-3}$ & reference sea water density \\
\hline$C_{p w}$ & $4000 \mathrm{~J} \mathrm{~K}^{-1} \mathrm{~kg}^{-1}$ & heat capacity of sea water \\
\hline$K_{h}$ & $200 \mathrm{~m}^{2} \mathrm{~s}^{-1}$ & horizontal heat diffusivity \\
\hline$A_{h x}$ & $2000 \mathrm{~m}^{2} \mathrm{~s}^{-1}$ & horizontal zonal eddy diffusivity \\
\hline$A_{h y}$ & $200 \mathrm{~m}^{2} \mathrm{~s}^{-1}$ & horizontal meridional eddy diffusivity \\
\hline$\beta$ & $1.6 \times 10^{-11} \mathrm{~m}^{-1} \mathrm{~s}^{-1}$ & $\partial f / \partial y$ at $45^{\circ} \mathrm{N}$ \\
\hline$R$ & $35 \mathrm{~km}$ & $\begin{array}{l}\text { first baroclinic Rossby radius of deformation } \\
\text { Coupling parameters }\end{array}$ \\
\hline$\lambda$ & $23 \mathrm{~W} \mathrm{~m}^{-2} \mathrm{~K}^{-1}$ & bulk heat transfert coefficient \\
\hline$\gamma$ & $2.4 \times 10^{-2} \mathrm{~m} \mathrm{~s}^{-1}$ & surface drag coefficient \\
\hline$r$ & 0.3 & fraction of latitude circle occupied by the ocean \\
\hline
\end{tabular}

Table 1: Standard values of the model parameters, as in Cessi (2000) except for the atmospheric eddy-diffusion $k_{s}$. 


\begin{tabular}{|c|c|c|c|c|c|c|c|c|c|c|c|}
\hline Exp & $\begin{array}{c}\mathrm{BC} \\
\text { free/no } \\
\text { slip }\end{array}$ & $\begin{array}{c}k_{s} \\
\left(\mathrm{~m}^{2} \mathrm{~s}^{-1}\right)\end{array}$ & $\begin{array}{c}A_{h x} \\
\left(\mathrm{~m}^{2} \mathrm{~s}^{-1}\right)\end{array}$ & $\begin{array}{c}A_{h y} \\
\left(\mathrm{~m}^{2} \mathrm{~s}^{-1}\right)\end{array}$ & $\begin{array}{c}K_{h} \\
\left(\mathrm{~m}^{2} \mathrm{~s}^{-1}\right)\end{array}$ & $\begin{array}{c}f \\
\text { lat. } \\
\left({ }^{\circ} \mathrm{N}\right)\end{array}$ & $\begin{array}{c}\gamma \\
\left(\mathrm{cm} \mathrm{s}^{-1}\right) \\
\end{array}$ & $\begin{array}{l}\mathrm{Nx} \\
\mathrm{Ny}\end{array}$ & $\begin{array}{c}\mathrm{KE} \\
\text { mean } \\
\left(\mathrm{J} \mathrm{m}^{-2}\right) \\
\end{array}$ & $\begin{array}{c}\mathrm{KE} \\
\text { st.dev. } \\
\left(\mathrm{J} \mathrm{m}^{-2}\right)\end{array}$ & $\begin{array}{c}\text { Oscil. } \\
\text { period } \\
(\mathrm{yr})\end{array}$ \\
\hline 1 & $\partial_{y} \tau=0$ & $2 \times 10^{6}$ & 2000 & 200 & 200 & 45 & 2.4 & 500 & 415.6 & 8.2 & 18.2 \\
\hline 2 & $\tau=0$ & $2 \times 10^{6}$ & 2000 & 200 & 200 & 45 & 2.4 & 200 & 612.0 & 3.8 & 21.4 \\
\hline 3 & $\tau=0$ & $2 \times 10^{6}$ & 2000 & 200 & 200 & 22 & 2.4 & 200 & 816.9 & 33.6 & 23.2 \\
\hline 4 & $\tau=0$ & $2 \times 10^{6}$ & 2000 & 200 & 200 & 33 & 2.4 & 200 & 683.8 & 20.2 & 19.2 \\
\hline 5 & $\tau=0$ & $2 \times 10^{6}$ & 2000 & 200 & 200 & 56 & 2.4 & 200 & 588.8 & 5.2 & 19.6 \\
\hline 6 & $\tau=0$ & $2 \times 10^{6}$ & 2000 & 200 & 200 & 67 & 2.4 & 200 & 560.9 & 2.3 & 16.8 \\
\hline 7 & $\tau=0$ & $2 \times 10^{6}$ & 2000 & 200 & 200 & 45 & 2.0 & 200 & 504.1 & 2.7 & 21.6 \\
\hline 8 & $\tau=0$ & $2 \times 10^{6}$ & 2000 & 200 & 200 & 45 & 2.8 & 200 & 715.1 & 5.3 & 21.8 \\
\hline 9 & $\tau=0$ & $2 \times 10^{6}$ & 2000 & 200 & 200 & 45 & 3.2 & 200 & 815.4 & 7.9 & $\sim 21.1$ \\
\hline 10 & $\tau=0$ & $2 \times 10^{6}$ & 2000 & 200 & 200 & 45 & 3.6 & 200 & 915.9 & 17.5 & $\sim 13.3$ \\
\hline 11 & $\tau=0$ & $2 \times 10^{6}$ & 2000 & 200 & 100 & 45 & 2.4 & 200 & 692.4 & 17.7 & 18.2 \\
\hline 12 & $\tau=0$ & $2 \times 10^{6}$ & 2000 & 200 & 300 & 45 & 2.4 & 200 & 570.0 & 3.8 & 20.2 \\
\hline 13 & $\tau=0$ & $2 \times 10^{6}$ & 2000 & 200 & 500 & 45 & 2.4 & 200 & 507.6 & 1.4 & 18.4 \\
\hline 14 & $\tau=0$ & $2 \times 10^{6}$ & 2000 & 200 & 800 & 45 & 2.4 & 200 & 476.3 & 0 & - \\
\hline 15 & $\tau=0$ & $2 \times 10^{6}$ & 500 & 200 & 200 & 45 & 2.4 & 200 & 1000.2 & 13.6 & 18.0 \\
\hline 16 & $\tau=0$ & $2 \times 10^{6}$ & 1000 & 200 & 200 & 45 & 2.4 & 200 & 750.8 & 2.8 & 20.4 \\
\hline 17 & $\tau=0$ & $2 \times 10^{6}$ & 1500 & 200 & 200 & 45 & 2.4 & 200 & 660.6 & 3.5 & 21.0 \\
\hline 18 & $\tau=0$ & $2 \times 10^{6}$ & 2500 & 200 & 200 & 45 & 2.4 & 200 & 579.5 & 3.9 & 21.6 \\
\hline 19 & $\tau=0$ & $2 \times 10^{6}$ & 2000 & 100 & 200 & 45 & 2.4 & 200 & 1079.5 & 20.9 & $\sim 25.0$ \\
\hline 20 & $\tau=0$ & $2 \times 10^{6}$ & 2000 & 150 & 200 & 45 & 2.4 & 500 & 792.9 & 15.2 & 17.8 \\
\hline 21 & $\tau=0$ & $2 \times 10^{6}$ & 2000 & 250 & 200 & 45 & 2.4 & 200 & 506.4 & 4.1 & 19.6 \\
\hline 22 & $\tau=0$ & $2 \times 10^{6}$ & 2000 & 350 & 200 & 45 & 2.4 & 500 & 342.5 & 0 & - \\
\hline 23 & $\tau=0$ & $1.5 \times 10^{6}$ & 2000 & 200 & 200 & 45 & 2.4 & 300 & 626.7 & 23.2 & 19.4 \\
\hline 24 & $\tau=0$ & $2.5 \times 10^{6}$ & 2000 & 200 & 200 & 45 & 2.4 & 300 & 647.9 & 5.6 & 19.0 \\
\hline 25 & $\tau=0$ & $2.7 \times 10^{6}$ & 2000 & 200 & 200 & 45 & 2.4 & 300 & 640.7 & 1.5 & 16.8 \\
\hline 26 & $\tau=0$ & $3 \times 10^{6}$ & 2000 & 200 & 200 & 45 & 2.4 & 300 & 671.0 & 0 & - \\
\hline 27 & $\partial_{y} \tau=0$ & $2.7 \times 10^{6}$ & 2000 & 200 & 200 & 45 & 2.4 & 500 & 438.9 & 0 & - \\
\hline 28 & $\tau=0$ & $2 \times 10^{6}$ & 2000 & 200 & 200 & 45 & 2.4 & 999 & 1768.0 & 10.5 & 16.3 \\
\hline
\end{tabular}

Table 2: Summary of the numerical experiments. BC is the boundary condition for surface wind stress. Oscillation period is provided when available, and oscillation amplitude is measured through standard deviation of oceanic kinetic energy. 